\title{
Transient Modelling and Simulation of Gas Turbine Secondary Air System
}

\author{
Theoklis Nikolaidis $^{1 *}$, Haonan Wang ${ }^{1}$, Panagiotis Laskaridis ${ }^{1}$ \\ 1 Centre of Propulsion Engineering, Cranfield University, Cranfield, UK \\ * Corresponding author - t.nikolaidis@cranfield.ac.uk
}

\begin{abstract}
The behaviour of the jet engine during transient operation and specifically its secondary air system (SAS) is the point of this work. This paper presents a methodological approach to develop a fast, one-dimensional transient platform for preliminary analysis of the flow behaviour in gas turbine engines secondary air system. For this purpose, different elements of the system including rotating chamber, pipe, turbine blade cooling, orifice, and labyrinth seal are modelled in a modular form. The validity of the developed models for each component is checked against experimental/publicly available data. Then, using a flow network simulation approach, the secondary air system of a two-spool turbofan engine is modelled and simulated in transient mode. The coupling effect between volume packing and swirl are considered in the simulation, under two pre-defined scenarios for step and scheduled boundary condition variations. In the step-change scenario, the boundary conditions are changed instantly to represent the flow behaviour of the SAS under extreme operating conditions (i.e. shaft fracture, flameout, etc.). In the scheduled scenario, the boundary conditions vary linearly with time to represent the performance of the SAS under normal operating conditions (i.e. acceleration and deceleration). The key findings include the fact that, under normal engine operation, the flow in the SAS varies smoothly and converges much faster than the primary flow by around one magnitude. Thus, it is reasonable to use steady-state SAS model to simulate SAS flow behaviour under these conditions. However, under extreme conditions (e.g. flameout), which could induce an abrupt change in the primary airflow properties (pressure, temperature), reverse airflow or choking conditions in SAS may be observed. This could result in a malfunction of the SAS, inducing further damages to the engine.
\end{abstract}

Keywords: Secondary air system, transient modelling and simulation, gas turbine engines, extreme operating conditions effects. 


\section{Nomenclature}

\section{Abbreviations}

COT

Combustor Outlet Temperature

FSP Front Sump Pressurization

HPT High-Pressure Turbine

HPC High-Pressure

Compressor

OD Off Design

OPR Overall Pressure Ratio

PR Pressure Ratio

RR Rolls-Royce

SAS Secondary Air System

TET Turbine Entry

Temperature

\section{Symbol}

A

$A_{c} \quad$ Cross Section Area of The Cooling Passage

$A_{g} \quad$ External Blade

Surface Area

$B i_{t b c} \quad$ Bi Number of The

Coating

$B i_{b w} \quad$ Bi Number of The

Blade Wall

$B i_{\text {total }} \quad B i_{t b c}-\left(\frac{\varepsilon_{c}-\eta_{f}}{1-\varepsilon_{c}}\right) B i_{b w}$

c Clearance

$C_{d} \quad$ Discharge Coefficient

$C_{v} \quad$ Constant Volume

Specific Heat

$C_{w} \quad$ Flow Coefficient, $\frac{\dot{m}}{\mathrm{M} r_{2}}$

$D \quad$ Pipe/Nozzle Diameter

F Angular Flux
$f_{D} \quad$ Friction Factor

$h_{g} \quad$ Gas Heat Transfer Coefficient

$K_{c}$

$S t_{g}\left(\frac{C_{p, g}}{C_{p, c}}\right)\left(\frac{A_{g}}{A_{\mathrm{C}}}\right)$

$\mathrm{K}_{c o}$

$\mathrm{K}_{\varphi}$

$L$

$M_{d}$

$M_{S}$

$m_{c v}$

$\dot{m}$

$\mathrm{n}_{t}$

P

$p$

$Q_{\text {net }}$

Re

$R$

$r$

$\mathrm{r}_{1}$

$\mathrm{r}_{2}$

Re

$s$

$S t_{g}$

$\mathrm{T}$

$t$

V

$V_{c v}$

$\gamma$
Carry-Over Factor

$$
\mathrm{V}_{\varphi} / r^{n}
$$

Pipe/ Nozzle Length

Disk Moment

Shroud Moment

Mass in Cavity

Mass Flow Rate

Labyrinth Seal Teeth Number

Pressure

Teeth Distance

Heat Input Due to

Disc Windage And

Convection

Reynold's Number

Gas Constant

Radius

Inner Radius

Outer Radius

Reynold's Number

Shroud Width

Stanton Number

Temperature

Time/ Teeth

Thickness

Velocity

Cavity Volume

Specific Heat Ratio 


\begin{tabular}{|c|c|c|c|}
\hline$\varepsilon$ & $\begin{array}{l}\text { Pipe Surface Absolute } \\
\text { Roughness }\end{array}$ & $\Delta_{b w}$ & Blade Thickness \\
\hline$\varepsilon_{c}$ & $\begin{array}{l}\text { Convection Cooling } \\
\text { Effectiveness }\end{array}$ & & \\
\hline$\eta_{c}$ & Cooling Efficiency & \multicolumn{2}{|c|}{ Subscripts } \\
\hline \multirow{2}{*}{$\eta_{f}$} & \multirow{2}{*}{$\begin{array}{l}\text { Cooling Efficiency of } \\
\text { Blade with Film } \\
\text { Cooling }\end{array}$} & 1 & Upstream \\
\hline & & 2 & Downstream \\
\hline \multirow[t]{2}{*}{$\lambda$} & \multirow{2}{*}{$\begin{array}{l}\text { Through Flow } \\
\text { Parameter }\end{array}$} & $t$ & Total \\
\hline & & $b$ & Blade \\
\hline \multirow[t]{2}{*}{$\lambda_{b w}$} & \multirow{2}{*}{$\begin{array}{l}\text { Conductivity } \\
\text { Coefficient of Blade } \\
\text { Metal }\end{array}$} & $c$ & Coolant \\
\hline & & $d$ & disc \\
\hline \multirow[t]{3}{*}{$\lambda_{t b c}$} & \multirow{3}{*}{$\begin{array}{l}\text { Conductivity } \\
\text { Coefficient of Thermal } \\
\text { Barrier Coating }\end{array}$} & $f$ & Film \\
\hline & & $g$ & Gas \\
\hline & & in & Inlet \\
\hline$\mu$ & Viscosity & out & Outlet \\
\hline$\rho$ & Gas Density & $s$ & Static \\
\hline \multirow[t]{2}{*}{$\varnothing$} & \multirow{2}{*}{$\begin{array}{l}\text { Coolant Mass Flow } \\
\text { Ratio }\end{array}$} & $t b c$ & Thermal Barrier Coating \\
\hline & & $x$ & Axial Direction \\
\hline$\omega$ & Angular Velocity & $\varphi$ & Tangential Direction \\
\hline$\Delta$ & Difference & & \\
\hline \multirow[t]{2}{*}{$\Delta_{t b c}$} & Thermal Barrier & \multirow{2}{*}{\multicolumn{2}{|c|}{$\begin{array}{l}\text { Superscripts } \\
-\quad \text { Average }\end{array}$}} \\
\hline & Coatıng Inıckness & & \\
\hline
\end{tabular}

\section{Introduction}

The secondary air system (SAS) of a gas turbine engine ensures that the engine can operate safely and perform efficiently. The air is extracted from the main gas path (therefore, it does not contribute to the thrust or power generation directly) and it is diverted to serve different engine systems (e.g. turbine blade cooling, oil sump pressurization, bearing control loading, etc.). It is well known that SAS operation affects the engine performance. Therefore, it is of primary importance to model the effects of SAS during the preliminary design for engines. Different flow elements, which have been developed as independent modules, can be used to build typical secondary airflow branches in a gas turbine engine, such as the turbine blade cooling air, the oil sump pressurization air, the disc cavity ventilation air, the axial bearing load control, etc. However, from the performance perspective, there are always efficiency losses associated with SAS. Typically, for a modern turbofan engine, an increase of $11 \mathrm{~K}$ in turbine entry temperature is required to compensate $1 \%$ rise in secondary air mass flow to maintain 
the same level of thrust [1]. Thus, SAS should be designed meticulously to achieve its functions with the minimum amount of bleeding air [2].

The investigation on SAS analysis has been in wide interests for a long time. The mainstream is implementing appropriate flow network to represent the SAS and to analyse the flow behaviour in the network. In 1992, Kutz and Speer [3] firstly introduced the flow network method for gas turbine SAS modelling. The authors have considered heat transfer along the flow path, choking in the pipe, two-phase flow, reverse flow and several pipes connecting in series. Although the results obtained from this method was satisfactory, its complexity appears as a huge obstacle for preliminary analysis as fast-run codes are needed in an iterative procedure of the preliminary design. In 2009, Alexiou et al [4] developed a model with fewer flow conditions considered (i.e. two-phase flow) in an object-oriented environment that allows the creation of different configurations in a simple and flexible manner. Che et al. [5] proposed a unified component method to model the SAS and the main gas path together to save the effort for establishing an interface between them. Besides the traditional deterministic models, Brack et al [6] put forward a probabilistic model to account for the uncertainty in the SAS due to geometric variations from engine to engine and varied engine operational conditions. In addition to the 1-D network, there are many research studies on integrating 1-D/2-D/3-D models to account for thermomechanical effects on performance as well as irregular geometries [7-9].

The above-mentioned studies focused on steady-state performance simulations. However, the behaviour of SAS during transient conditions should be investigated, as several phenomena may take place, which cannot be captured during steady-state simulations. Simulating the SAS at transient conditions may detect and prevent short excess in axial bearing loading and helps to understand the system's dynamic characteristics under extreme conditions (e.g. when flameout, disc burst, or shaft fracture) [10].

Recently, several methods have been published on the modelling of SAS transient performance. Gallar [11] and Calcagni [12] have developed a SAS modelling platform, which has been successfully validated with SPAN, a Rolls-Royce (RR) in-house program for performing SAS calculation. The dynamic properties of the pipe have been modelled comprehensively in their work. However, the rotating effects of elements were not considered. In 2010, May and Chew proposed an initial method to account for the rotating effects in transient simulation [13]. They later improved the initial methods to simulate the transient performance of a de-swirl nozzle by considering more details of the swirl structure in the rotating chamber [14]. The swirl structure is utilized to assess the radial pressure distribution in the chamber and for a better thermal model. However, they focused on a standalone flow 
element of the chamber and did not extend their investigation to flow network simulation. Moreover, the effects on different operating conditions on the behaviour of SAS have not been considered yet.

In this paper, the coupling effect between volume packing and swirl is considered as a step further in SAS transient simulation. Besides, other elements were also developed to support a full-branch analysis of the flow network. At the same time, the platform is constrained to onedimensional for a faster calculation speed. Therefore, the main contribution of this work is the development of a fast, one-dimensional transient platform for preliminary analysis of the flow behaviour in the gas turbine engine SAS. The tool is utilized to simulate the transient performance under both normal and extreme conditions considering the coupling effect between volume packing and swirl in a full flow network.

For this purpose, section two describes the methodology and validation of the platform components. The different elements of the SAS system (i.e. rotating chamber, pipe, and turbine cooling passages, orifice, and labyrinth seal) are presented in this section. The developed models are validated against available experimental data. In section three, the flow network simulation for the different SAS branches (the front sump pressurization branch, highpressure turbine rotor cooling branch, and cavity ventilation branch) is demonstrated. In section four, the analysis and discussion of the results are presented, followed by the conclusion remarks in section five.

\section{Methodology and Validation of the Platform}

In this paper, the one-dimensional flow network method is used for the modelling of transient performance of the SAS. The flow elements in the transient modelling platform are categorised into volumes and flow path elements, which are connected to form the system network. The volumetric effects and gas inertia are considered for the volumes while the flow path elements just serve as inlet and outlet of the volumes.

The physical SAS transient process will take place once there is a deviation from equilibrium engine operating points, i.e. due to transient in the primary airflow system. The air mass flow and pressure change will affect the SAS inlet conditions and a new energy and mass flow balance should be achieved. Based on the physical process, the transient performance is modelled as follow: at each time step, the mass flow rate is firstly calculated from pressure imbalance at each flow element, and then the air temperature is calculated according to different heat transfer processes taking place at each flow element.

A typical gas turbine engine SAS can be modelled by using a library of five elements: a rotating chamber, a pipe and turbine cooling passages, which are classified as volumes; and an orifice, a labyrinth seal, which are treated as a flow path. After establishing the network from the flow 
elements, parameters specifying the element characteristics, boundary conditions, initial pressure and temperature within the chamber should be provided to initialize the calculation. The chamber's pressure, temperature and the mass flow rate on each branch will be calculated to satisfy the boundary conditions. The mathematical model and the corresponding assumptions made for each element are explained in the following sections.

\subsection{Element modelling}

The mathematical modelling of different components of the SAS system will be presented in this section.

\subsubsection{Modelling of the rotating chamber}

A schematic of a rotating chamber is shown in Figure 1. The inlet flow could be injected radially (Figure 1 (a)) or axially (Figure 1 (b)). The direction could have an impact on the vortex structure in the chamber. It is assumed that the chamber is in constant size, rectangular shape and the gas properties keep uniform in the axial direction.

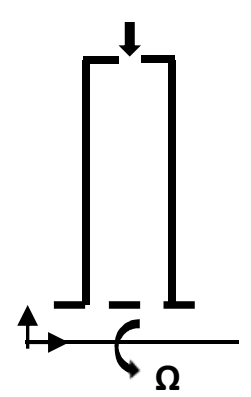

(a)

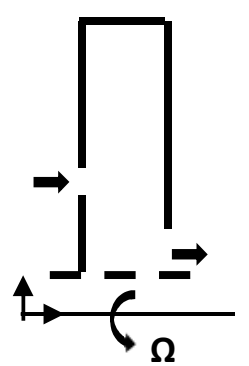

(b)

Figure 1: Schematic of a rotating chamber [21]:

(a) with radially inward flow; (b) with axially inward flow

The mathematical modelling has been based on the work from May et al in [13-14]. However, in [13-14], the convection heat transfer with the surroundings was not considered. Its inclusion in this model is based on the work of Alexiou et al [4].

It is assumed that the tangential velocity profile satisfies:

$$
\mathrm{V}_{\varphi}=\mathrm{K}_{\varphi} * \mathrm{r}^{\mathrm{n}}
$$

where $\mathrm{V}_{\varphi}$ presents tangential velocity; $\mathrm{K}_{\varphi}$ is a constant; $\mathrm{r}$ is the radius and $\mathrm{n}$ is vortex index, indicating the vortex behaviour in the chamber. Equation (1) shows that the tangential velocity varies exponentially along the radius. When $n$ equals to 1 , the tangential velocity of the flow in the chamber maintains a forced vortex profile; when $n$ equals to -1 , a free vortex structure 
will be observed in the flow. For axial inflow from any radius, the vortex structure is assumed to be forced vortex, which means vortex index $n$ should be 1 . For radial inflow, it is assumed that the vortex index could be determined by the through-flow parameter $\lambda[14]$ :

$$
\lambda=\frac{C_{w}}{R e^{0.8}}
$$

where

$$
C_{w}=\frac{\dot{m}}{\mu r_{2}}
$$

where $C_{w}$ represents the flow coefficient; $\mu$ is the viscosity; $\dot{m}$ the mass flow rate; $r_{2}$ represents the outer cavity radius and $R e$ is Reynold's number.

According to [10], the relationship between vortex index $n$ and through-flow parameter $\lambda$ could be estimated as:

$$
n=\left\{\begin{array}{l}
1, \quad \text { if }-0.001<\lambda<0.01 \\
-1, \quad \text { if } \lambda<-1 \text { or } \lambda>0.4
\end{array}\right.
$$

However, when $\lambda$ is outside of the range (i.e. $-1<\lambda<-0.001$ or $0.01<\lambda<0.4$ ), there is no determinant relationship in this transition area. It may be assumed that it varies exponentially or linearly [10]. A linear relationship is assumed in this paper for simplicity (Figure 2).

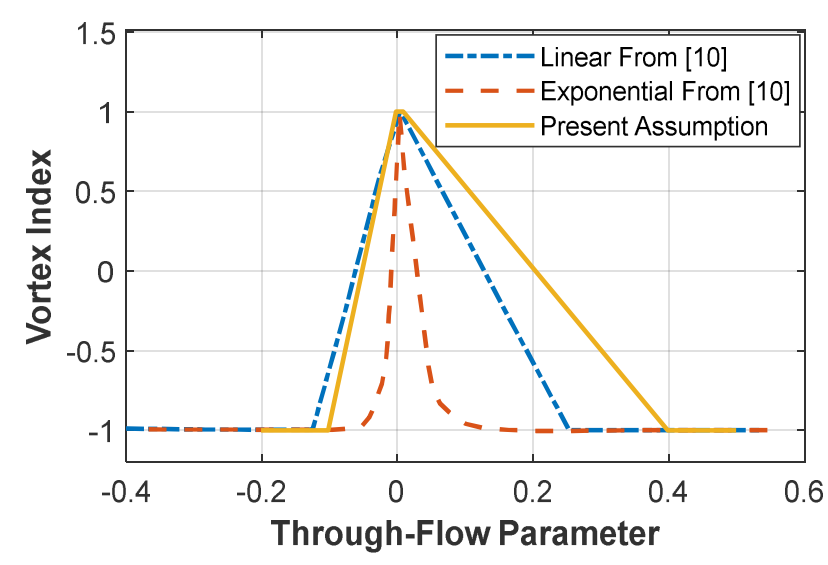

Figure 2: Vortex index vs. through flow parameter

The rate of chamber average static pressure change can be obtained from the mass conservation law:

$$
\frac{d \overline{P_{s}}}{d t}=\frac{R \overline{T_{s}}}{V_{c v}} \cdot\left(\dot{m}_{\text {in }}-\dot{m}_{\text {out }}\right)+\frac{\overline{P_{s}}}{\overline{T_{s}}} \frac{d \overline{T_{s}}}{d t}
$$


where $V_{c v}$ represents the constant cavity volume, $R$ is the gas constant and $\overline{P_{S}}$ and $\overline{T_{s}}$ are the average chamber static pressure and temperature respectively. It should be noted that, in this model the swirl structure is used for calculating the disc windage and radial pressure distribution is not considered for a faster calculation.

The rate of chamber static temperature change can be calculated from energy conservation law:

$\frac{d \overline{T_{s}}}{d t}=\frac{1}{m_{c v}\left(\bar{T} \frac{d C_{v}}{d \bar{T}}+C_{v}\right)}\left[\dot{m}_{\text {in }} C_{p, \text { in }} T_{t, \text { in }}-\dot{m}_{\text {out }} C_{p, \text { out }} T_{t, \text { out }}-C_{v} \bar{T}_{s}\left(\dot{m}_{\text {in }}-\dot{m}_{\text {out }}\right)+Q_{\text {net }}\right]$

The kinetic energy variation has been neglected in equation (5), and it is assumed that the mass flow out of the chamber at average temperature and pressure. $Q_{\text {net }}$ in the equation (5) consists of the work input due to disc windage and convection heat transfer from the disc. Conduction through the disc wall has not been considered in this model. Determination of these values can be found in Appendix equations $(A-11)$ to $(A-13)$. The swirl velocity $V_{\varphi}$ can be obtained from angular momentum conservation law:

$$
\frac{d}{d t}\left(\rho V_{\varphi}\left(\frac{1}{n+3}\right)\left(\frac{r_{2}^{n+3}-r_{1}^{n+3}}{r_{2}^{n}}\right)\right)=\frac{1}{2 \pi s}\left(\sum M_{d}+\sum M_{s}-F_{\text {out }}+F_{\text {in }}\right)
$$

where $r_{1}$ and $r_{2}$ represents the inner and outer cavity radius respectively, $s$ is the shroud width, $M_{d}$ and $M_{s}$ are the disc moment and shroud moment respectively. Their estimation can be found in the Appendix equations $(A-1)$ to $(A-10)$. F represents the angular flux entering and exiting the cavity:

$$
\begin{gathered}
F_{\text {out }}=\dot{m}_{\text {out }} K_{\varphi} r_{\text {out }}^{n+1} \\
F_{\text {in }}=\dot{m}_{\text {in }} V_{\varphi, \text { in }} r_{\text {in }}
\end{gathered}
$$

\subsubsection{Modelling of the pipe}

A pipe is modelled as a rotating chamber with a large length to volume ratio. In the 1-D pipe model, due to the small radius of it, the angular momentum effect is not considered. The basic mass, momentum and energy conservation laws are similar to that of the chamber (equation (4)-(6)). Darcy-Weisbach's formula has been selected to model the total pressure loss [25]:

$$
\Delta \mathrm{P}=L \cdot f_{D} \cdot \frac{\rho}{2} \cdot \frac{V^{2}}{D}
$$


where $\Delta \mathrm{P}$ represents the total pressure loss; $L$ is the pipe length; $\rho$ is air density; $V$ represents the flow velocity in the pipe; $\mathrm{D}$ is the pipe diameter and $f_{D}$ is the friction factor, which is determined using Moody's formula [25]:

$$
f_{D}=0.0055\left[1+\left(2 \times 10^{4} \cdot \frac{\varepsilon}{D}+\frac{10^{6}}{R e}\right)^{1 / 3}\right]
$$

where $\varepsilon$ represents the absolute roughness of pipe surface and $R e$ is the Reynold's number of the air.

\subsubsection{Modelling of the turbine blade cooling}

In the turbine blade cooling model, there are two processes: the pumping and heat transfer process, which are considered separately. Initially, the process in the cooling passage is assumed adiabatic. Given this, a pipe element is used to model the pumping effect in order to calculate the coolant mass flow rate, which is available in the cooling passage. The next step is to enable the heat transfer model and evaluate the blade temperature. In other words, the blade temperature is calculated based on instant coolant mass flow and combustor outlet temperature (COT). These two distinct processes do not compromise the accuracy of the results considerably, as the flow pressure does not influence the heat transfer in the classical analytical blade cooling model.

The restricting effect through the coolant passage is the same as the pipe analyzed in the previous section. For the thermal effects, a semi-empirical model is implemented for the preliminary analysis of the blade cooling effects [15]. The assumptions used for this model are the uniform bulk gas around blade, uniform external heat transfer coefficients, constant thermodynamic properties and uniform metal temperature. Some technical empirical data should be provided to obtain the blade metal temperature, which are shown in Table 1.

Table 1: Empirical technical values input for turbine blade cooling element

\begin{tabular}{|c|c|c|}
\hline Symbol & Meaning & Definition \\
\hline$B i_{t b c}$ & $\begin{array}{c}\text { Bi number of the thermal } \\
\text { barrier coating }\end{array}$ & $B i_{t b c}=\frac{h_{g} \Delta_{t b c}}{\lambda_{t b c}}$ \\
\hline$B i_{b w}$ & Bi number of the blade wall & $B i_{b w}=\frac{h_{g} \Delta_{b w}}{\lambda_{b w}}$ \\
\hline$\eta_{f}$ & $\begin{array}{c}\text { Cooling efficiency of blade } \\
\text { with film cooling }\end{array}$ & $\eta_{f}=\frac{T_{g}-T_{f}}{T_{g}-T_{c, \text { out }}}$ \\
\hline$\eta_{c}$ & Cooling efficiency & $\eta_{c}=\frac{T_{c, o u t}-T_{c, \text { in }}}{T_{b}-T_{c, \text { in }}}$ \\
\hline
\end{tabular}




\begin{tabular}{|l|l|l|}
\hline$S t_{g}$ & Stanton number & $S t_{g}=\frac{h_{g}}{\left(\dot{m}_{c} / A_{c}\right) C_{p, g}}$ \\
\hline
\end{tabular}

Given the coolant mass flow ratio $\emptyset$, which can be obtained from the SAS calculation, the convection cooling effectiveness $\varepsilon_{c}$ can be solved from the following equation:

$$
\varnothing=\frac{\dot{m}_{c}}{\dot{m}_{g}}=\frac{K_{c}}{1+B i_{\text {total }}} \frac{\varepsilon_{c}-\eta_{f}\left[1-\eta_{c}\left(1-\varepsilon_{c}\right)\right]}{\eta_{c}\left(1-\varepsilon_{c}\right)}
$$

where $K_{c} B i_{\text {total }}$ can be calculated as:

$$
\begin{gathered}
K_{c}=S t_{g}\left(\frac{C_{p, g}}{C_{p, c}}\right)\left(\frac{A_{g}}{A_{c}}\right) \\
B i_{\text {total }}=B i_{t b c}-\left(\frac{\varepsilon_{c}-\eta_{f}}{1-\varepsilon_{c}}\right) B i_{b w}
\end{gathered}
$$

where the definition $S t_{g}, \eta_{f}, B i_{t b c}, B i_{b w}$ can be found in Table $1 ; C_{p, g}$ is the specific heat capacity of the hot gas and $C_{p, c}$ is the specific heat capacity of the coolant; $A_{g}$ is the external blade surface area which can be estimated as the product of the blade perimeter and the blade span; and $A_{c}$ is the cross sectional area of the coolant passage.

Given $\varepsilon_{c}$, the blade temperature can be obtained from the equation:

$$
\varepsilon_{c}=\frac{T_{g}-T_{b}}{T_{g}-T_{c, i n}}
$$

where $T_{g}$ represents the TET and it can be extracted from the main path calculation; $T_{c, i n}$ represents the inlet coolant temperature which can be obtained from SAS calculation system.

\subsubsection{Modelling of the orifice}

The flow behavior of the orifice is assumed to be similar to that of a nozzle. A typical formula depicting the characteristic of the nozzle is used to model the orifice:

$$
\dot{m}=C_{d} \cdot A \cdot \rho_{t, 1} \cdot\left(\frac{P_{s, 2}}{P_{t, 1}}\right)^{\frac{1}{\gamma}} \cdot\left\{\frac{2 \gamma}{\gamma-1} \cdot \frac{P_{t, 1}}{\rho_{t, 1}} \cdot\left[1-\left(\frac{P_{s, 2}}{P_{t, 1}}\right)^{(\gamma-1) / \gamma}\right]\right\}^{0.5}
$$

where $C_{d}$ is the discharge coefficient, $A$ represents the nozzle area, $\rho_{t, 1}$ is the upstream total density, $P_{t, 1}$ represents the upstream total pressure, $P_{s, 2}$ is the downstream static pressure and $\gamma$ represents the fluid adiabatic index.

The rotating effects are accounted for modifying the discharge coefficient. Besides, it is assumed that the heat transfer effect can be neglected for the modelling of the orifice. The determination of the discharge coefficient $C_{d}$ can be found in the Appendix equations $(A-15)$ to $(A-24)$. 
Moreover, flow reversal and choking have been considered in the orifice element in this study. Given the upstream total pressure and downstream static pressure, the algorithm would check firstly if the pressure ratio is larger than one. If so, it indicates that the downstream static pressure is larger than upstream pressure, and the air would flow reversely. The flow function is:

$$
\dot{m}=-C_{d} \cdot A \cdot \rho_{t, 1} \cdot\left(\frac{P_{s, 2}}{P_{t, 1}}\right)^{-\frac{1}{\gamma}} \cdot\left\{\frac{2 \gamma}{\gamma-1} \cdot \frac{P_{t, 2}}{\rho_{t, 2}} \cdot\left[1-\left(\frac{P_{s, 2}}{P_{t, 1}}\right)^{-(\gamma-1) / \gamma}\right]\right\}^{0.5}
$$

And then, the algorithm would check if the pressure ratio is larger than the critical value to determine whether the orifice is choked. If it's choked, the pressure ratio used to drive the air is the critical one:

$$
P R_{\text {crit }}=\left(1+\frac{\gamma-1}{2}\right)^{\frac{\gamma-1}{\gamma}}
$$

\subsubsection{Modelling of the labyrinth seal}

A schematic of the labyrinth seal is shown in Figure 3 with critical parameters annotated in the figure. Where $t$ indicates thickness, $p$ represents teeth distance and $c$ indicates clearance.

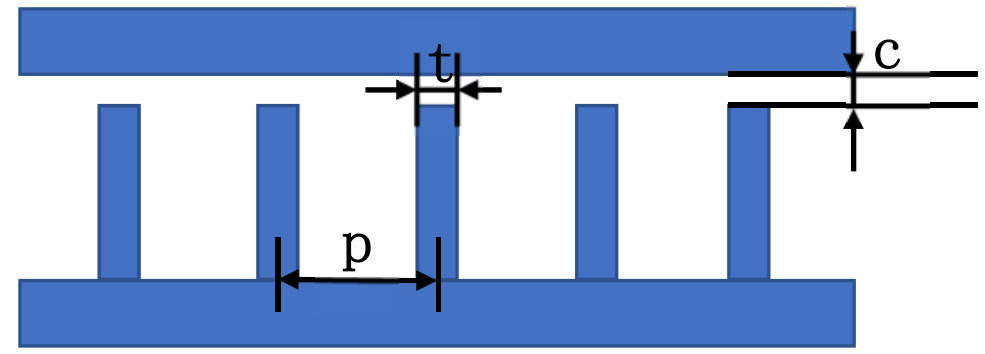

Figure 3 Schematic of a labyrinth seal

A classical mathematical equation is used to model the labyrinth seal [19]:

$$
\dot{\mathrm{m}}=C_{d} \cdot \mathrm{K}_{c o} \cdot \mathrm{A} \cdot \frac{\mathrm{P}_{\mathrm{t}, 1}}{\sqrt{\mathrm{R} \cdot \mathrm{T}_{\mathrm{t}, 1}}} \cdot \sqrt{\frac{1-\left(\frac{\mathrm{P}_{s, 2}}{\mathrm{P}_{\mathrm{t}, 1}}\right)^{2}}{\mathrm{n}_{t}+\ln \frac{\mathrm{P}_{\mathrm{t}, 1}}{\mathrm{P}_{s, 2}}}}
$$

where A represents the annular area between the fin tip and the casting, $\mathrm{n}_{t}$ is the teeth number and $\mathrm{K}_{c o}$ is the carry-over factor representing the flow resistance of the labyrinth teeth.

The value of discharge coefficient $\left(C_{d}\right)$ can be chosen to 0.71 when the clearance-to-thickness ratio $(\mathrm{c} / \mathrm{t})$ is between 1.3 and 2.3 [24]. The determination of carry-over factor $\left(\mathrm{K}_{c o}\right)$ is based on the clearance-to-distance ratio $(c / p)$ and teeth number $\left(n_{t}\right)$. Its specific formula is provided in 
the Appendix equation (A-14). The consideration of flow reversal and choking is done in a similar way to the orifice model described in the previous sub-section.

\subsection{Element Validation and Parametric Study}

In order to confirm the validity of the modelling approach, some elements have been validated against experimental/publicly available data.

\subsubsection{Validation of the rotating chamber}

The first test is to demonstrate the coupling effect of disc windage moments and vortex structure for a chamber with radially inward flow. A schematic display of the rotating chamber is shown in Figure 1 (a). The inner radius of the chamber is $0.1 \mathrm{~m}$ and the outer radius is 0.5 $\mathrm{m}$. The hypothetical fluid ( $\rho=10 \mathrm{~kg} / \mathrm{m}^{3}, \mu=10^{-5} \mathrm{~Pa} \cdot \mathrm{s}$ ) is assumed to enter the chamber from the outer radius at half-disc rim speed. Then, the cumulative moment and tangential velocity profile along the radius direction is recorded as vortex index $(n)$ changes from -1 to 1 , where $\mathrm{K}$ and $C_{m, \text { disc }}$ in Appendix equation (A-5) are set to be 1 and 0.002 respectively.

The results showed that both parameters (cumulative moment $\mathrm{M}$ and tangential velocity profile $V_{\varphi}$ ) align perfectly with those published in [14] (Figure 4 and Figure 5 respectively).

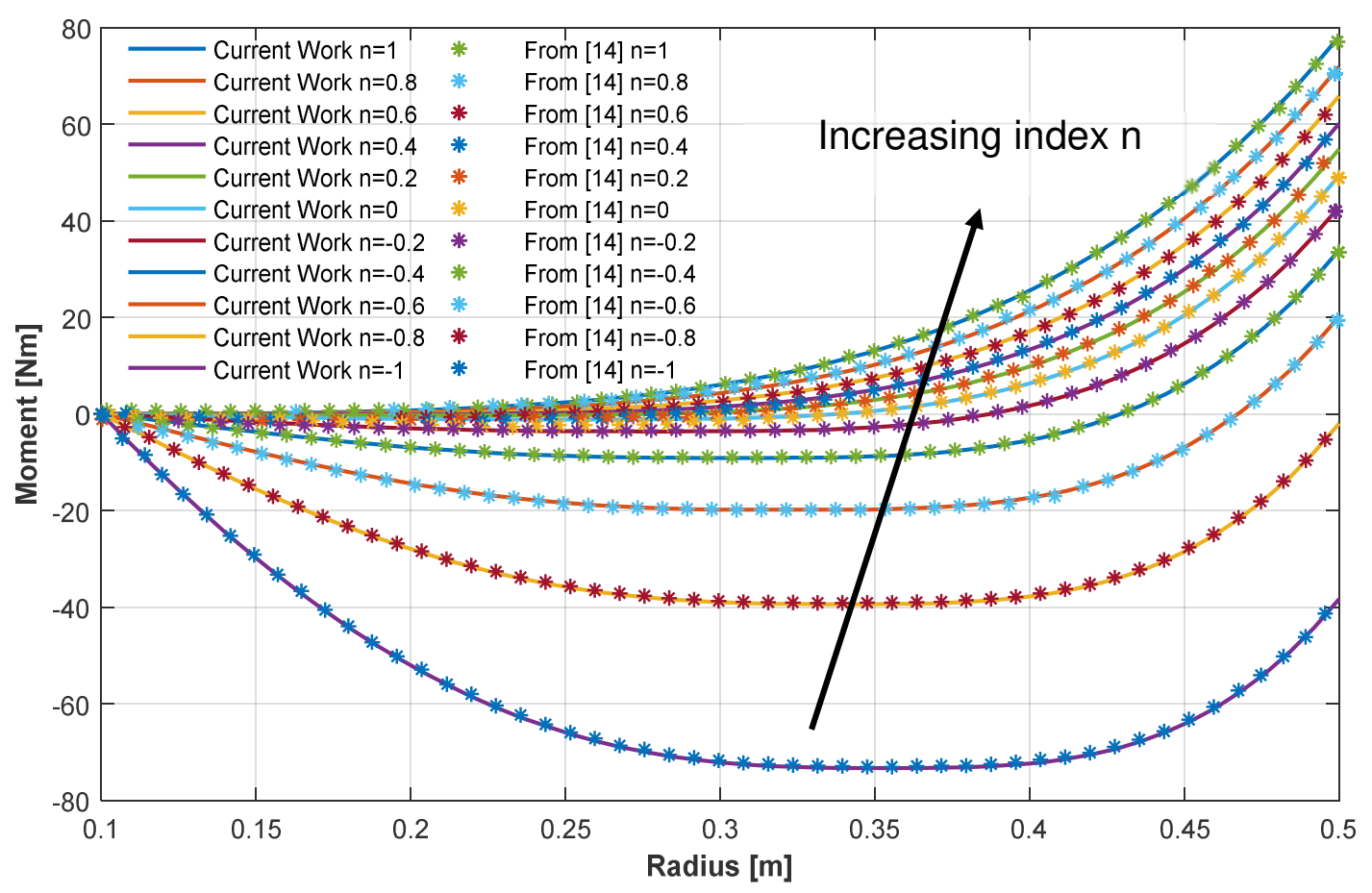

Figure 4: Cumulative moment profile. 


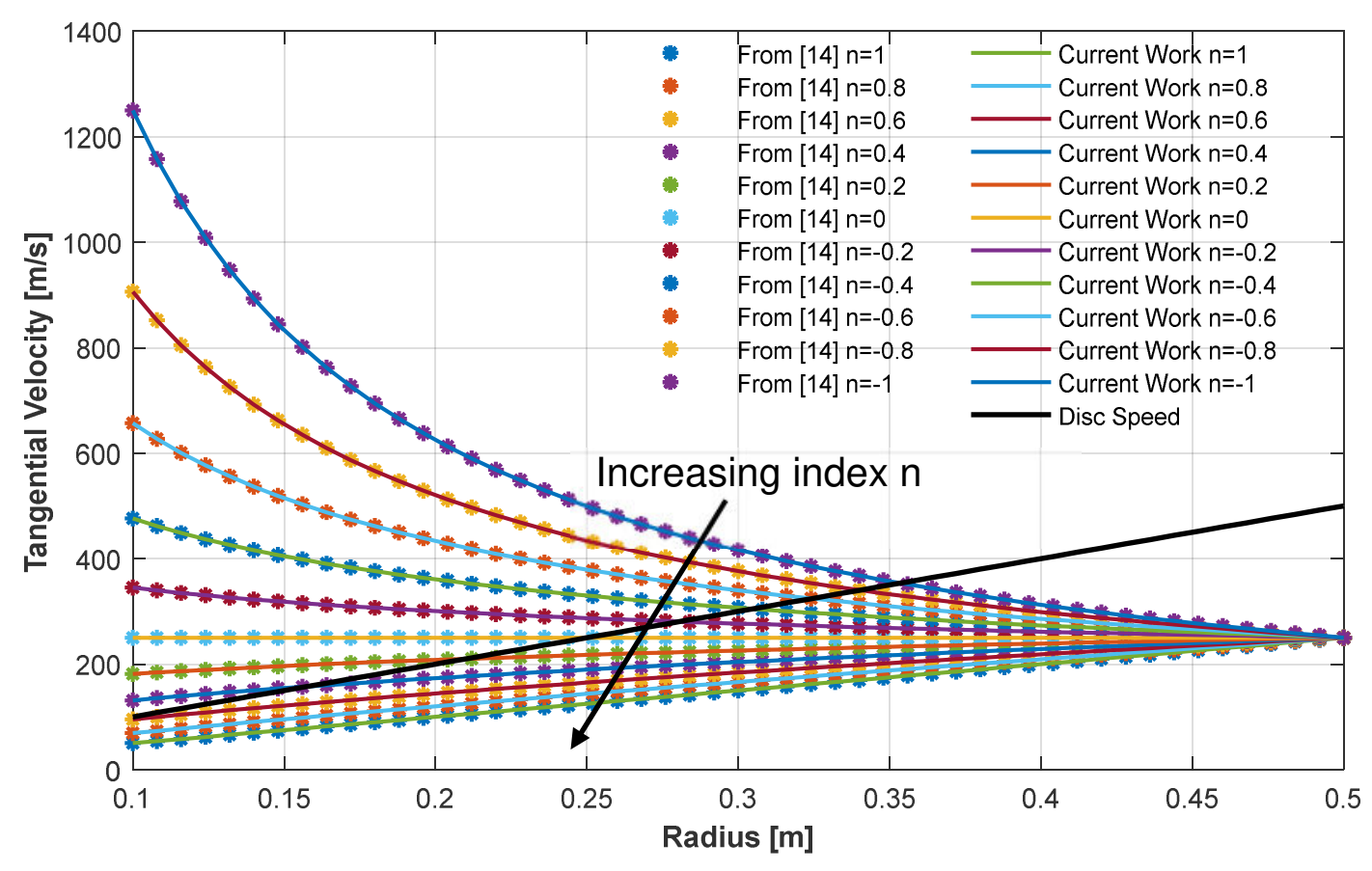

Figure 5: Tangential velocity profile.

The second test is to demonstrate the dynamic characteristics of the air in a chamber with axially inward flow, as shown in Figure 2(b). The air is assumed to be driven into the chamber from the inner radius $0.0284 \mathrm{~m}$ and exit from the outer radius $0.5 \mathrm{~m}$. The test conditions are shown in Table 2 [13]. The chamber was at equilibrium state with initial inlet pressure to be $689.476 \mathrm{kPa}$, then a $10 \%$ increase at the inlet pressure was imposed while keeping the exit pressure and inlet temperature fixed at $482.633 \mathrm{kPa}$ and $500 \mathrm{~K}$ respectively to analyze the dynamic flow behavior in the chamber.

The original results recorded in [14] only present the response in the first $0.1 \mathrm{~s}$. To compare the outcome after $0.1 \mathrm{~s}$ (i.e. up to $0.16 \mathrm{~s}$ ), it is assumed that the system is settled down after $0.1 \mathrm{~s}$ (dotted line in figure 6). For the current work, the settle down time was found to be around $0.12 \mathrm{~s}$, which is $20 \%$ longer than the time in [14]. The settled volume average static pressure is $586.054 \mathrm{kPa}$, which is in a good agreement with experimental results reported in [14], where the relative error during the transient process is within $2 \%$ and the steady state error (i.e. settle down period) is around $1.2 \%$ (Figure 6 ). 
Table 2: Test conditions for validation of the chamber under transient characteristic

\begin{tabular}{|l|c|}
\hline \multicolumn{2}{|c|}{ Initial conditions } \\
\hline \multicolumn{1}{|c|}{ Parameter } & Value \\
\hline Initial inlet pressure $\left(P_{\text {in }}\right)[\mathrm{kPa}]$ & 689.476 \\
\hline Inlet pressure step size $(\Delta P)[\mathrm{kPa}]$ & 68.9476 \\
\hline Inlet temperature $\left(T_{\text {in }}\right)[\mathrm{K}]$ & 500 \\
\hline Exit pressure $\left(P_{\text {out }}\right)[\mathrm{kPa}]$ & 482.633 \\
\hline Rotational speed $\left(\omega_{d}\right)[\mathrm{rpm}]$ & 10000 \\
\hline Inlet area $\left(A_{\text {in }}\right)\left[\mathrm{m}^{\wedge} 2\right]$ & 0.0014 \\
\hline Outlet area $\left(A_{\text {out }}\right)\left[\mathrm{m}^{\wedge} 2\right]$ & 0.0025 \\
\hline
\end{tabular}

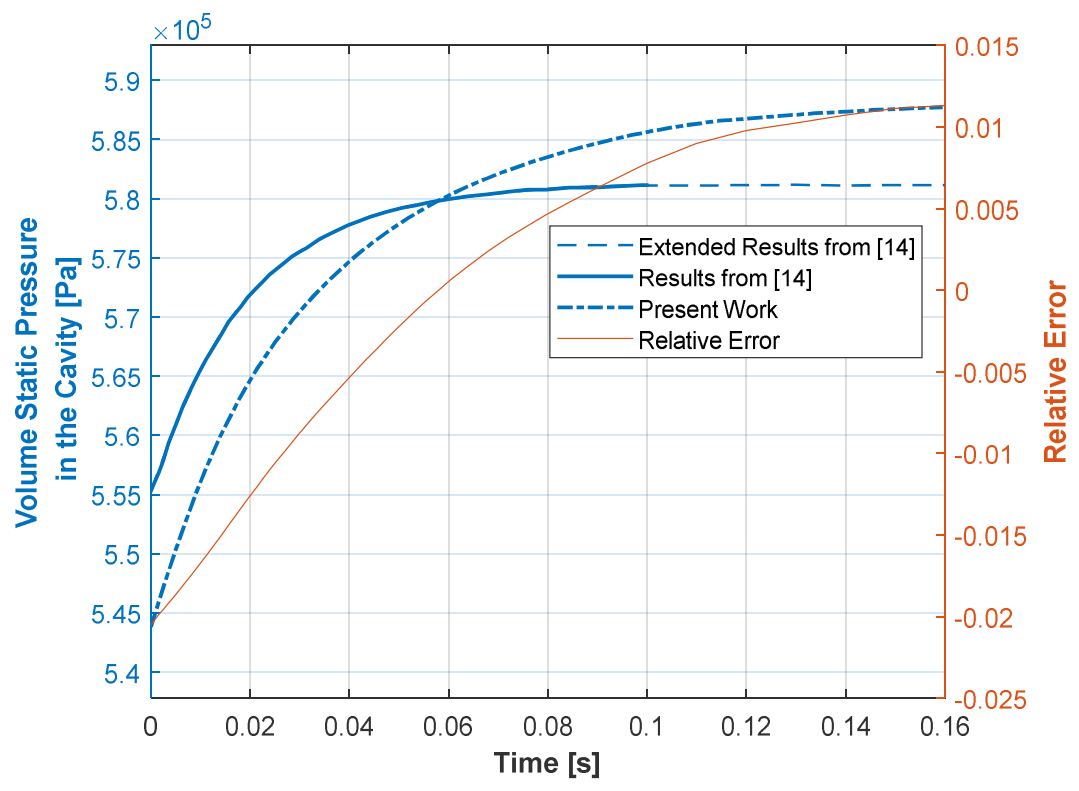

Figure 6: Volume average static pressure response.

The performance of the flow in the pipe is similar to that of the chamber since similar mathematical model implemented.

\subsubsection{Parametric study of the turbine blade cooling model}

In order to investigate the behavior of the turbine blade cooling model, the upstream conditions kept fixed (with Pin set to $114.595 \mathrm{kPa}$, Tin set to $359 \mathrm{~K}$, TET set to $1700 \mathrm{~K}$ ) and a change in the downstream static pressure was imposed (varying from $68.757 \mathrm{kPa}$ to $114.595 \mathrm{kPa}$ ) to evaluate the variation of flow properties. The results show that the parameters follow the 
expected trends. The corrected mass flow rate increases with the rise of pressure ratio and the blade temperature drops because of the increase in coolant mass flow (Figure 7).

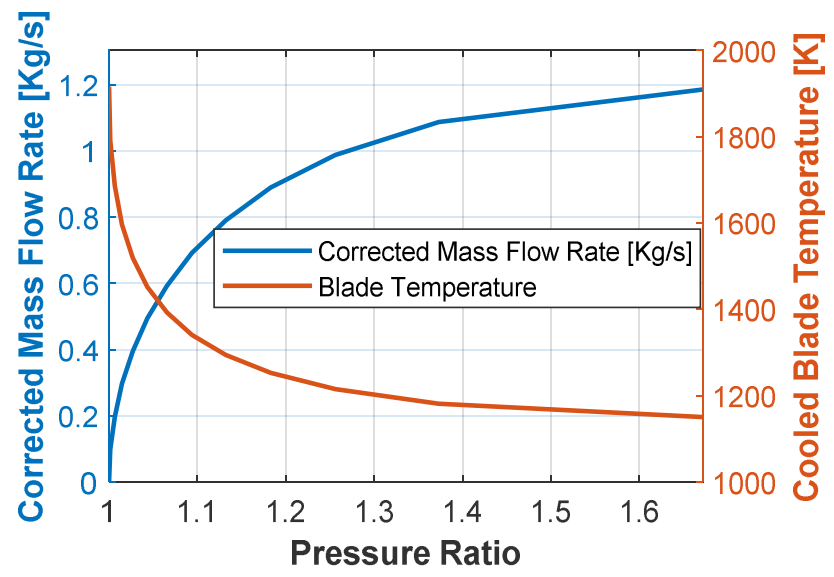

Figure 7: Parametric study of turbine blade cooling model

2.2.3 Parametric study of the labyrinth seal and orifice

The characteristics of the orifice and labyrinth seal were tested by keeping the upstream pressure fixed (Pin= $114.595 \mathrm{kPa}$ and $314.595 \mathrm{kPa}$ respectively) and changing the downstream pressure from $50 \%$ to $200 \%$ of the upstream pressure to evaluate the impact of pressure ratio on mass flow. It shows that transient orifice can handle reverse flow satisfactorily (Figure 8). When the pressure ratio is less than one, the flow is reversed (negative values), while it is not the case when the pressure ratio is higher than one. Obviously, when it equals 0 , there is no flow.

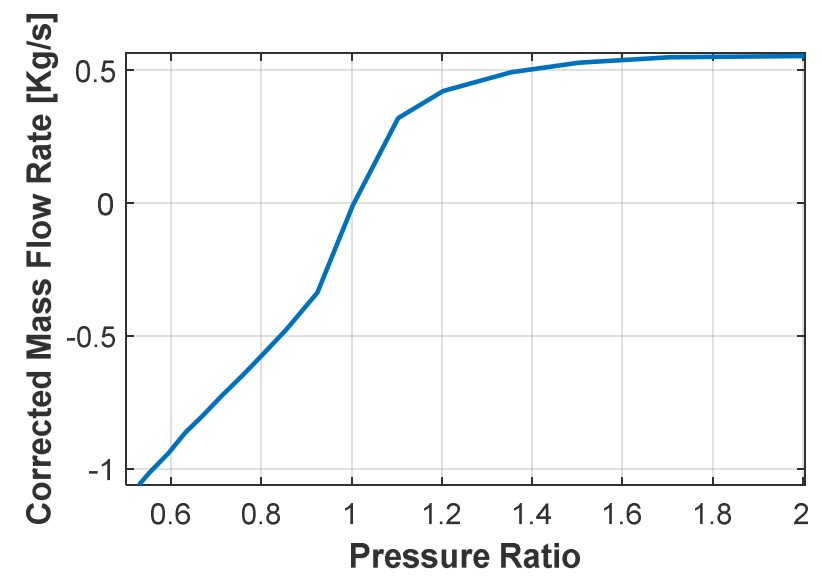

Figure 8: Orifice corrected mass flow rate response with pressure ratio

Choking conditions are indicated by the choking indicator parameter. Its value varies between 0 and 1.0 with the latter to indicate that choke occurs. In this case study, this happened when 
the pressure ratio was larger than 1.89 or smaller than 0.53 , which is consistent with the physical case (Figure 9).

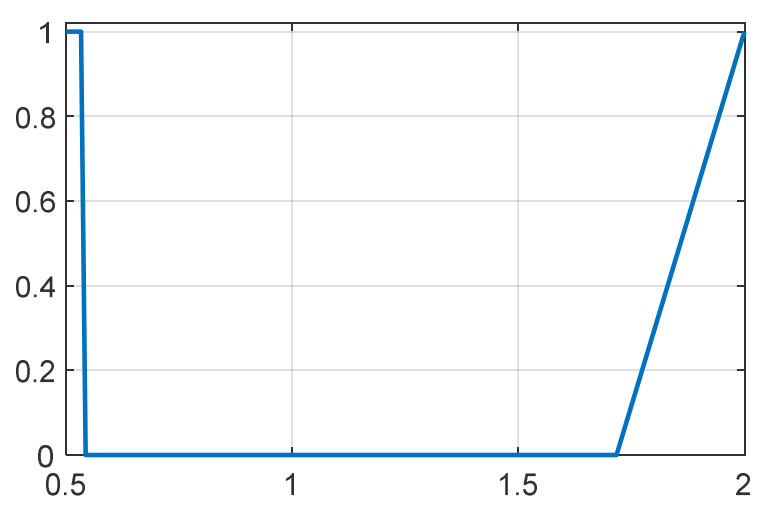

Figure 9: Orifice choking indicator

Finally, Figure 10 shows the characteristics of the labyrinth seal and its capability to deal with reverse flow. Similar to the orifice model, the non-dimensional mass flow becomes negative when the pressure ratio is less than one.

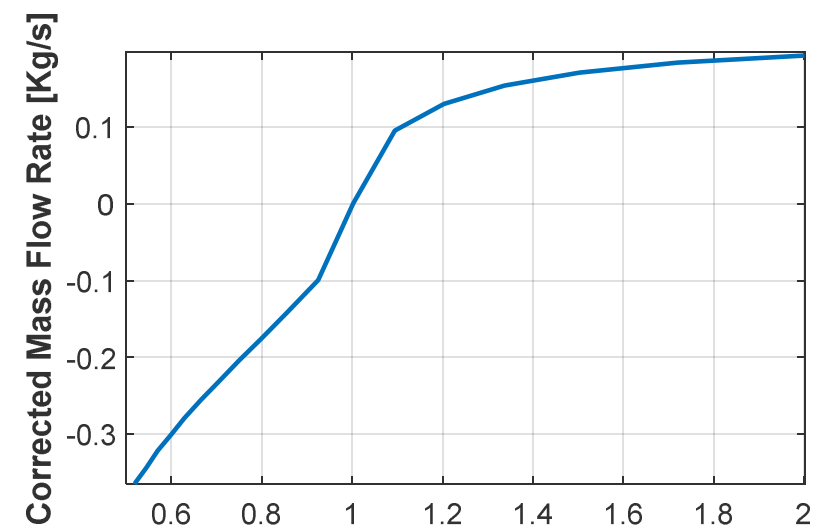

Figure 10: Labyrinth seal corrected mass flow rate response with pressure ratio

\section{Flow Network Simulation}

The described components can be used in a modular fashion in order to simulate a given SAS. As a case study, the SAS of a 2-spool turbofan engine (inspired by CFM56-7B) was built and tested in order to confirm the validity and capabilities of the platform in generating reasonable results. 


\subsection{Model set up}

Engine performance was simulated using Turbomatch, an in-house 0-D performance simulation code, developed at Cranfield University and featuring OD and transient simulations [22-23]. The boundary conditions for the SAS were extracted from the whole engine simulation.

In this engine model, the SAS is used for turbine blade cooling, cavity ventilation, oil sump pressurization, and customer bleeding. These functions are modelled through the seven branches with the flow passages marked in black as shown in Figure 11. The flow branch models are developed based on the cross-section view of the engine [16], relevant information [17] and the authors' engineering judgement.

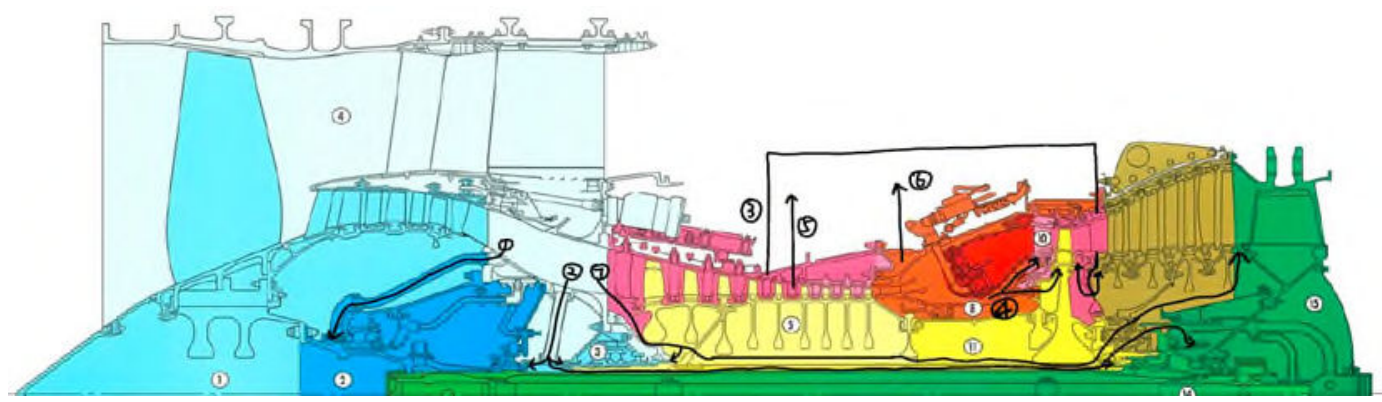

Figure 11: SAS model - engine photo from [16]

Three branches have been simulated in the platform: branch (1) (front sump pressurization), branch (4) (HPT rotor cooling), and branch (7) (cavity ventilation). As the flow structure of Front Sump Pressurization (FSP) and mid \& rear sump pressurization are similar (since all the air is tapped from the booster discharge and driven through a passage to achieve the oil sump and finally constrained in the oil chamber by the labyrinth seal), only FSP has been modelled to show the dynamic performance of the pipe. HPT rotor cooling branch is simulated, especially to test the dynamic characteristics of the blade cooling model and the possible overshooting in blade metal temperature. Ventilation air has been modelled to test the dynamic characteristics of chambers in series. Appropriate components from the developed library have been chosen to simulate the corresponding flow elements in the SAS. They are indexed in each category (e.g. orifice, labyrinth seal etc.) and indicated in different colors in Figure 12. 

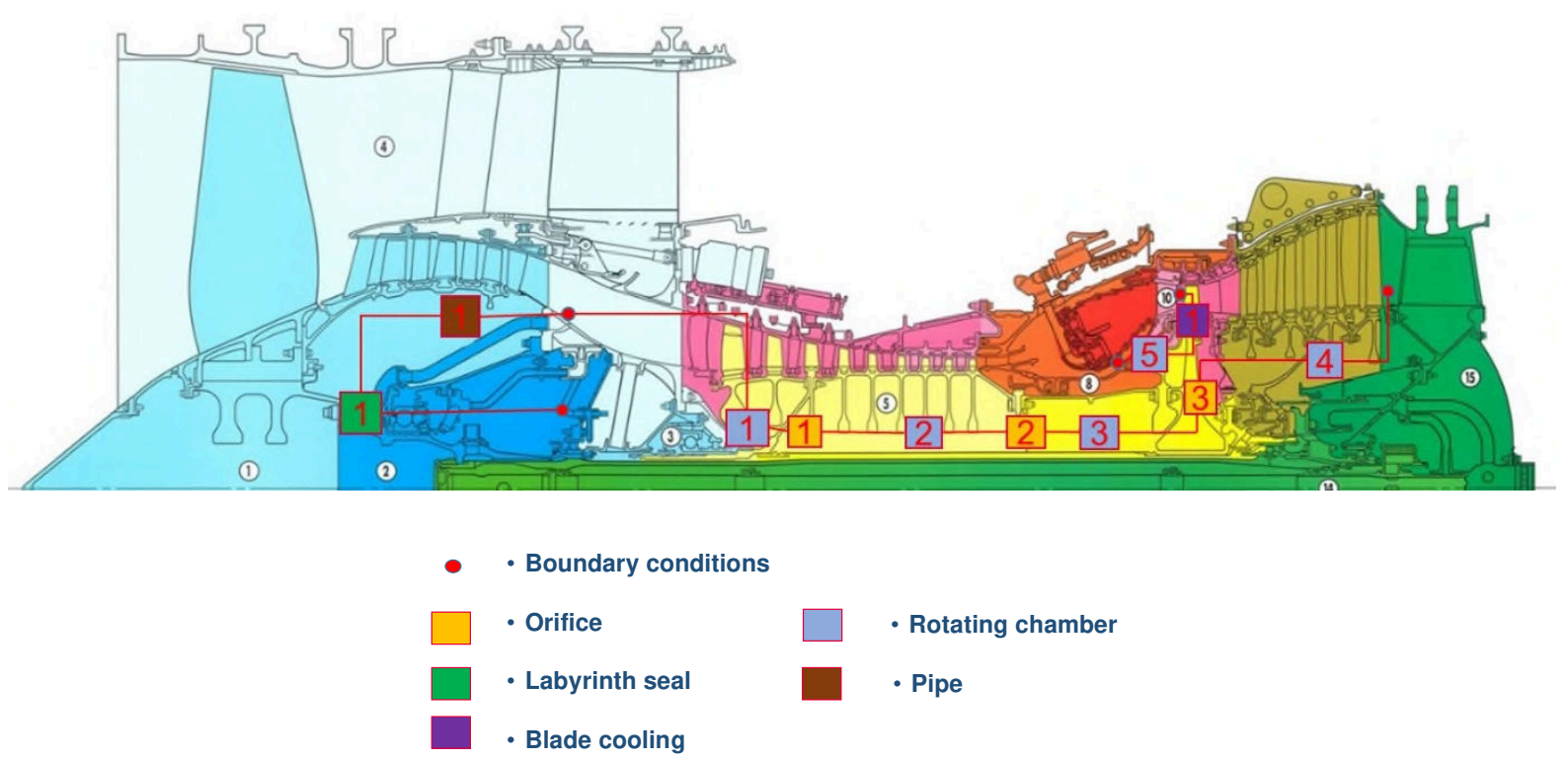

Figure 12: Model set up scheme - engine photo from [16]

A diagram representing the SAS integrating with the primary airflow is also shown in Figure 13.

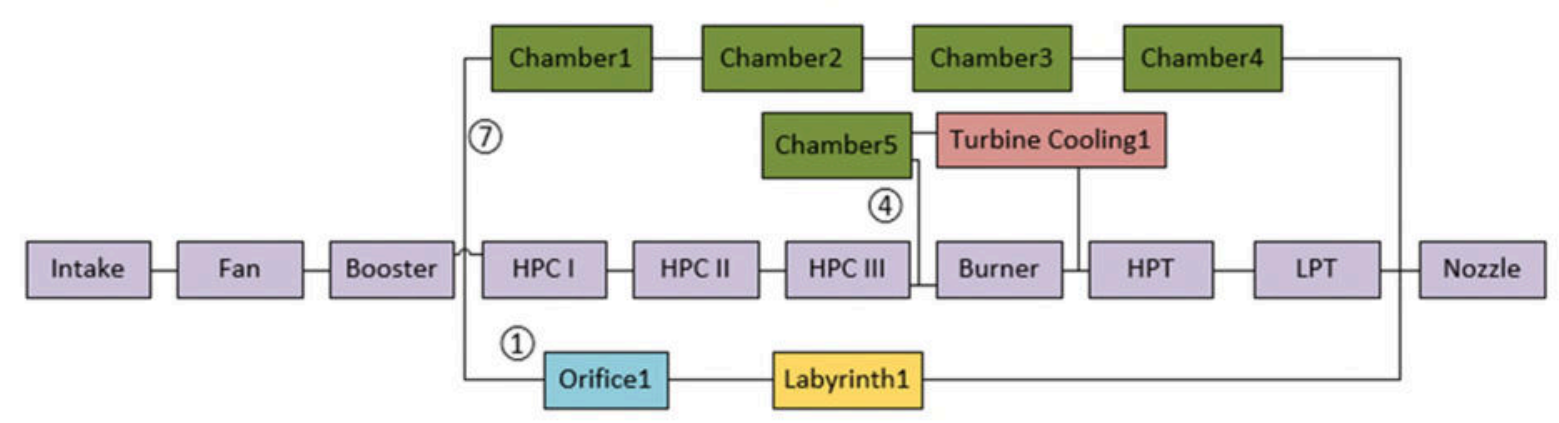

Figure 13: SAS flow network diagram

\subsection{Test definition}

Two transient cases are defined to test the behaviour of the designed system at both normal and extreme working conditions.

- The first case (I) simulates the flow behaviour under extreme working conditions (e.g. flameout) by introducing a step-change in the P \& T boundary conditions.

- The second case (II) simulates the flow behaviour under normal engine operation (e.g. acceleration and deceleration) by introducing a scheduled, linear and smooth variation of the boundary conditions.

\subsubsection{Case I: Extreme operating condition}

This case simulates the flow when there is a step-change in the boundary conditions. In the beginning, the SAS is in equilibrium state at the maximum operating condition, and then, the 
boundary conditions suddenly change to their minimum values. The two conditions are distinguished by the fuel flow to represent flameout situation, as shown in Table 3, and the corresponding boundary conditions for SAS are shown in Table 4.

Table 3: Test engine operation condition range

\begin{tabular}{c|c|c|c|c} 
Test Index & Altitude $(\mathrm{m})$ & Mach & Delta $T(\mathrm{~K})$ & Fuel flow $(\mathrm{kg} / \mathrm{s})$ \\
\hline \hline Initial & 0 & 0 & 15 & 1.2285 \\
Final & 0 & 0 & 15 & 0.41813
\end{tabular}

Table 4: step change of the boundary conditions

\begin{tabular}{|c|c|c|c|c|c|}
\hline \multirow{2}{*}{} & \multirow{2}{*}{ Branch } & \multicolumn{2}{|c|}{ Inlet } & \multicolumn{2}{c|}{ Outlet } \\
\cline { 3 - 6 } & & $\begin{array}{c}\text { Pressure } \\
(\mathbf{P})[\mathrm{kPa}]\end{array}$ & $\begin{array}{c}\text { Temperature } \\
(\mathbf{T})[\mathrm{K}]\end{array}$ & $\begin{array}{c}\text { Pressure } \\
(\mathbf{P})[\mathrm{kPa}]\end{array}$ & $\begin{array}{c}\text { Temperature } \\
(\mathbf{T})[\mathrm{K}]\end{array}$ \\
\hline \multirow{2}{*}{ Initial } & $(1)$ & 310.0545 & 434 & 149.961 & 827 \\
\cline { 2 - 6 } & $(4)$ & 3120.81 & 876 & 2999.22 & 1680 \\
\hline \multirow{2}{*}{ Final } & $1(7)$ & 176.3055 & 362 & 112.4708 & 659 \\
\cline { 2 - 6 } & $(4)$ & 1459.08 & 691 & 1398.285 & 1240 \\
\hline
\end{tabular}

\subsubsection{Case II: Normal operating condition}

The scheduled transient test simulates the expected operation of the SAS when there is a smooth change in the boundary conditions. The initial and final values of the engine operating conditions are the same as case one, shown in tables 3 and 4 . The variation of the boundary conditions represents the transient performance of the primary air system during deceleration. 
The boundary conditions vary linearly from the initial to the final value (table 4 ) in 3 seconds as shown in figure 14 (normalised values in the y-axis).

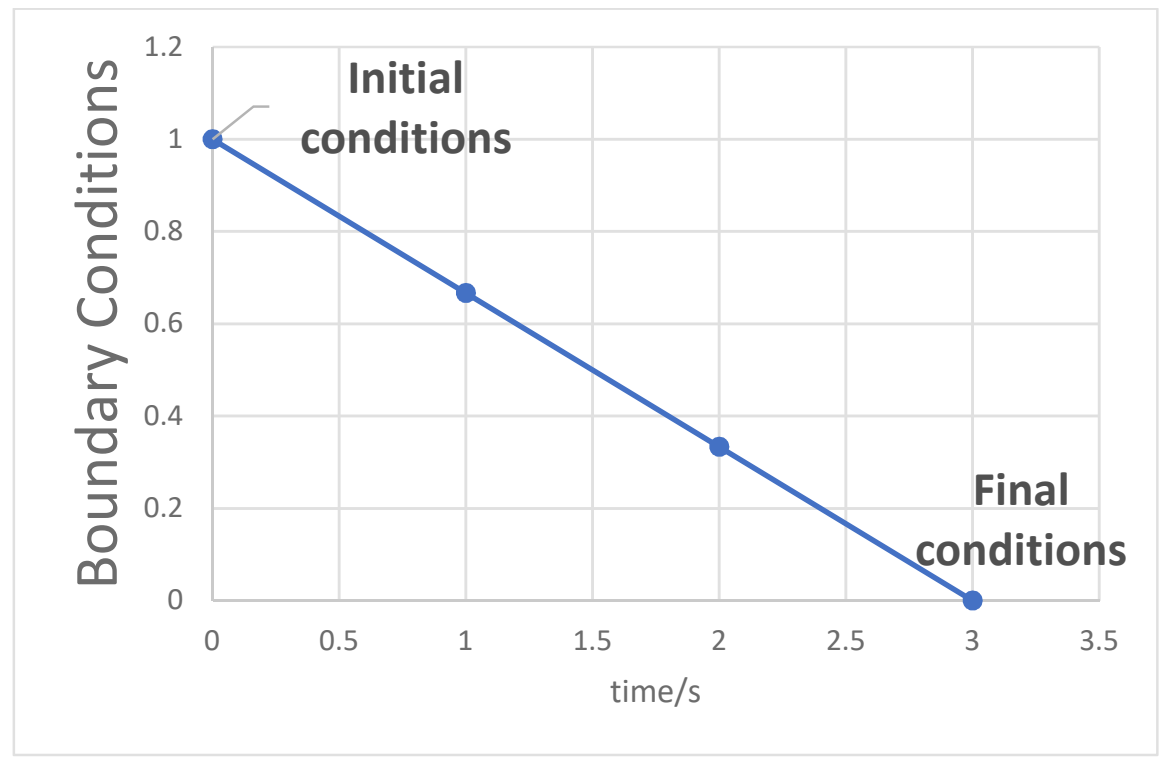

Figure 14: Schedule of the boundary condition variation

\section{Results analysis and Discussion}

The scenarios defined above have been simulated and the analysis of the results for the three branches are presented in this section. It should be noted that the convergence criterion is the equilibrium of SAS static pressure and primary air total pressure at the intersections between SAS and primary airflow, i.e. the bleed tap-off and exit ports of SAS. This is a simplification as the calculation of primary air static pressure requires the values of flow angles and crosssectional areas for those bleed ports. However, this assumption does not compromise the validity of the method.

\subsection{Case I: Extreme operating condition}

\subsubsection{Front Sump Pressurization (FSP) Branch Results}

In FSP (Figure 13 - branch 1), once the transient commences, the pipe $\mathrm{T}$ decreases dramatically due to the fast change of the heat flux, resulting from the quick variation of the mass flow. In the first $0.01 \mathrm{~s}$, the temperature reduces at $390 \mathrm{~K}$ (Figure 15). The curve flattens out due to the longer settling time for the angular momentum to reach convergence. The pipe temperature settles down after around $0.2 \mathrm{~s}$. It's a lot larger than the settling time of the pressure (Figure 16), that's because the pressure wave transmits in the air at the speed of sound, while the heat transfer rate is controlled by Newton's law for convection and angular momentum conservation law for disc windage work input, which is insignificant compared with speed of sound. The pressure at the pipe outlet can reach the new equilibrium point within 
$0.002 \mathrm{~s}$ (Figure 16). Therefore, equilibrium is achieved quite faster than the primary air system. The convergence time depends heavily on the geometry of the system. The larger the inlet and outlet area comparing to the chamber volume, the smaller the time constant, but oscillation may occur at the same time.

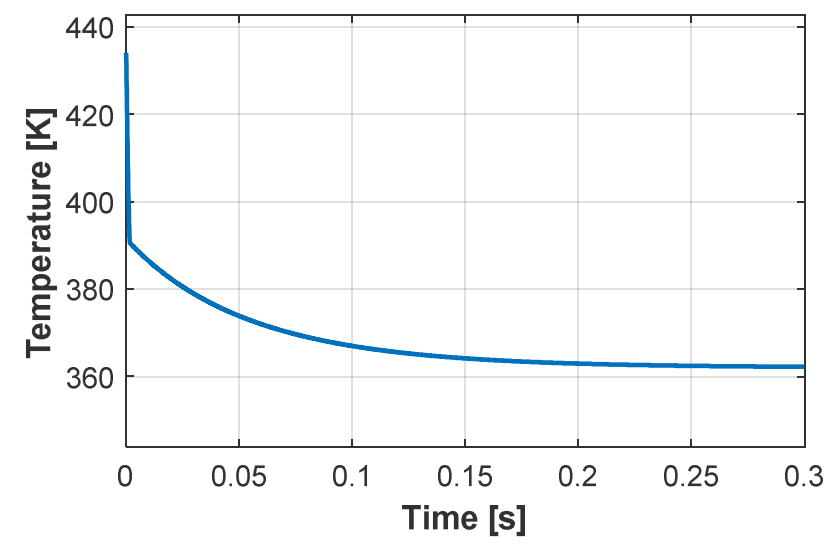

Figure 15: Average total temperature response in the cooling pipe in FSP under extreme conditions

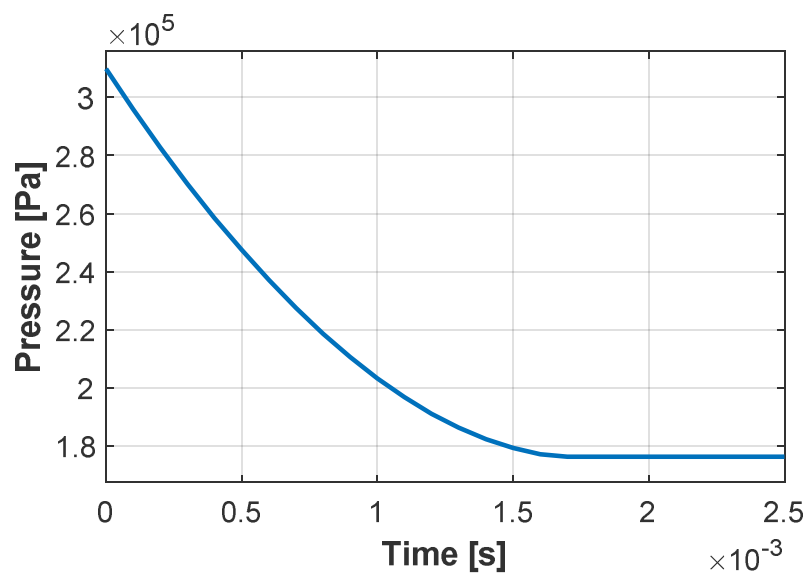

Figure 16: Average static pressure response in the cooling pipe in FSP under extreme conditions

\subsubsection{Ventilation Branch Results}

In the cavity ventilation branch (Figure 13 - branch 7), there are 4 chambers in series. Considerable reversed flow is noticed due to the sudden change of boundary conditions (i.e. negative values of mass flow rate - Figure 17). In figure 17, the mass flow response is plotted for the exit planes of the different rotating chambers and the entrance for the first chamber. 


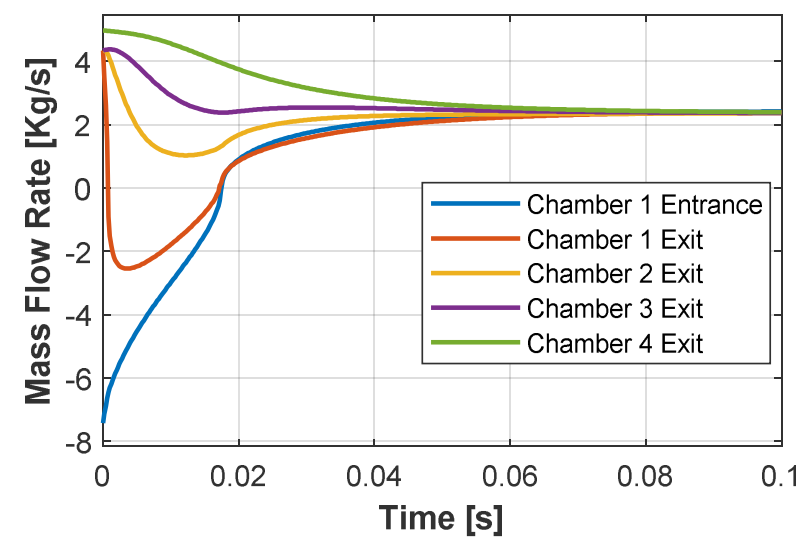

Figure 17 Average mass flow rate response in ventilation branch under extreme conditions

The reverse flow condition is noticeable in the upstream chamber (i.e. the chamber at the front end of HPC). That means that hotter gases flow from the back end HPC to the front end of HPC, affecting the disks' temperature. During the transient process, it can be observed that a large amount of air is expelled from SAS through the first chamber entrance and last chamber exit, indicated by a large flow reversal at the front plane and a large positive flow at the backplane. In general terms, SAS, in this case, acts like a high-pressure source, which drives its air out to the primary air system. Although the process takes place, the large flow reversal could result in a "fast pressure loading" process. In that case, SAS would serve as a lowpressure sink, and the much hotter air from the primary air system would be ingested into SAS, causing damages to discs and other components within SAS.

The pressure response in this flow branch explains the behaviour of the mass flow rate (Figure 18). Initially, the chambers' pressure is at a steady state. When the upper boundary pressure drops dramatically to a value of $176.305 \mathrm{kPa}$, which is considerably smaller than the initial pressure of the first chamber (around $3120.81 \mathrm{kPa}$ ), it results in a large flow reversal at the entrance plane. This creates a sharp pressure drop in the first chamber, and its exit pressure is smaller than the pressure in the downstream chamber 2 , and results in flow reversal at the first chamber exit. For chamber 3 and 4, due to the large pressure drop at the downstream boundary, the positive pressure ratio increases a lot and result in large positive mass flow rate. In addition to this, the settling time in this branch is around $0.08 \mathrm{~s}$, much larger than the FSP branch due to the greater volumetric effects. 


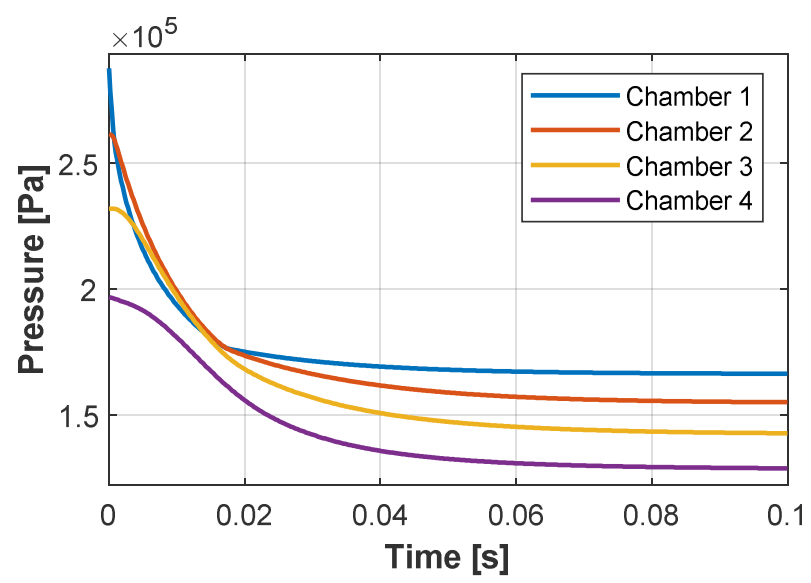

Figure 18: Average chamber static $\mathrm{P}$ response in ventilation branch under extreme conditions

The variation of T during transient depends mainly on the heat flux due to the mass flow rate (Figure 19). Thus, it shows a similar trend as the net mass flow increases in each chamber. And the overall decreasing trending again proves the analogue that SAS is like a highpressure source under this "fast pressure relief" situation.

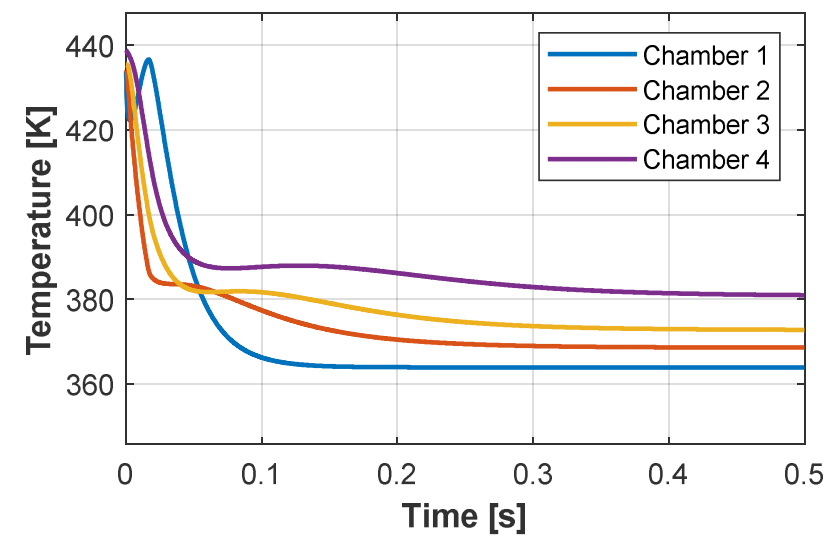

Figure 19: Average chamber total T response in HPT branch under extreme conditions

\subsubsection{HPT rotor blade cooling branch results}

The HPT rotor blade cooling branch (Figure 13 - branch 4) is modelled with a pre-swirl nozzle and cooling passage. The $P$ response in the cooling passage is much faster than that in the pre-swirl nozzle due to the different geometry configurations (Figure 20). In terms of the T, it takes roughly $0.5 \mathrm{~s}$ for the temperature to stabilize in this case (Figure 21).

Figure 22 shows the response of HPT rotor blade metal temperature after cooling. Since inertia of the metal temperature is not accounted for in this paper, the calculated blade temperature is based on instant coolant mass flow and COT. A noticeable undershoot is observed at the beginning $(<0.01 \mathrm{~s}$, figure 22$)$. It can be argued that the blade is safe in this 
case. However, when it is under the opposite conditions, i.e. "fast pressure loading" situation, this undershoot can become an overshoot, increasing the risk of failure.

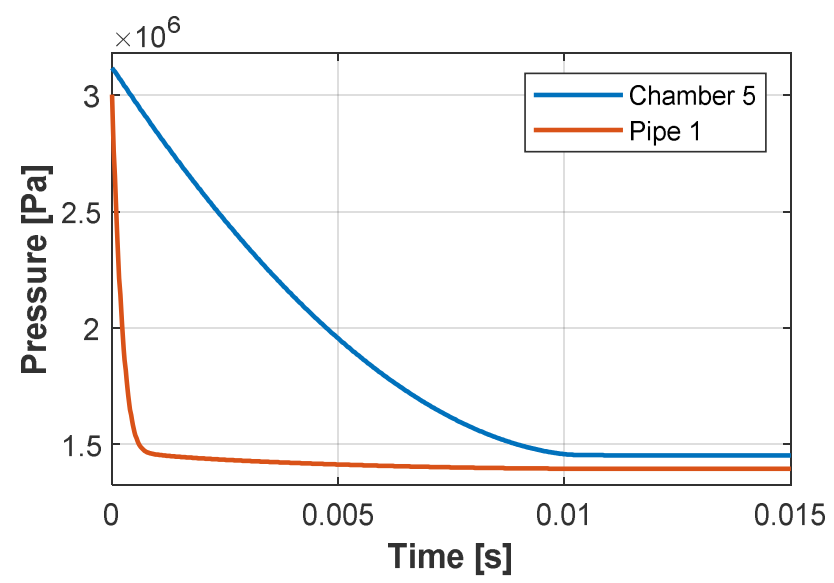

Figure 20: Average static $\mathrm{P}$ response in HPT branch under extreme conditions

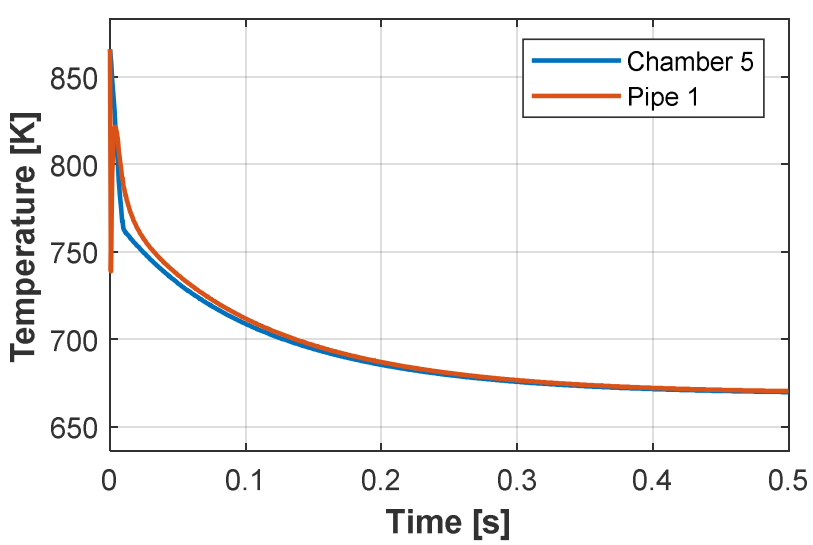

Figure 21: Average total T response in HPT branch under extreme conditions

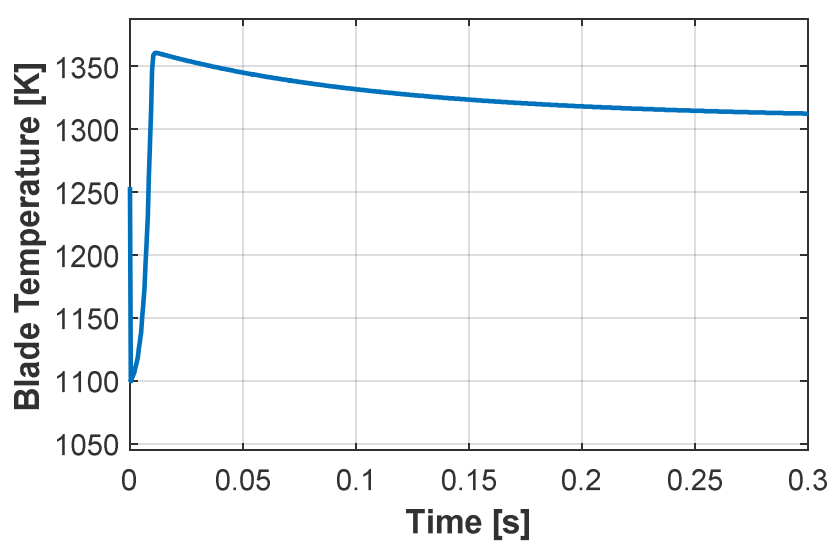

Figure 22 Blade temperature response in HPT branch under extreme conditions 


\subsection{Case II: normal operating condition}

In this case, there is a smooth transition of the boundary conditions described in section 3.2.2. The initial and final values of the engine operating conditions are the same as case one, shown in tables 3 and 4 . It was found that the SAS flow characteristics follow a smooth pattern. T and $P$ follow the changes in the boundary conditions for all the branches (Figures 23-25). In this case, no flow reversal or choking problem was observed. The blade temperature also varies in a mild way (Figure 26). Therefore, it is sufficient to use steady-state SAS simulate techniques rather than transient under normal engine operations.

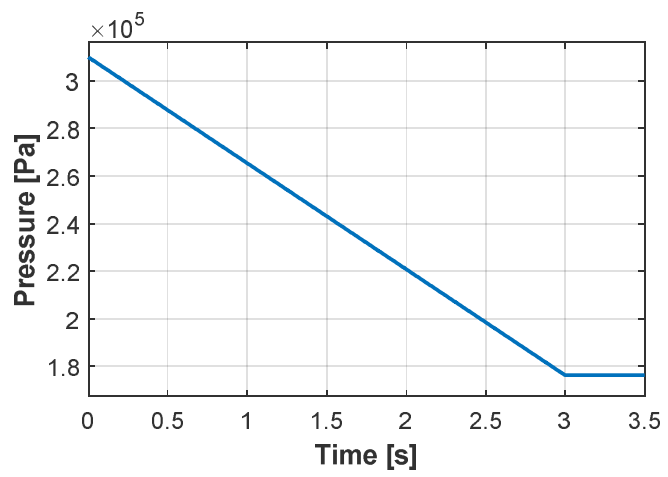

(a)

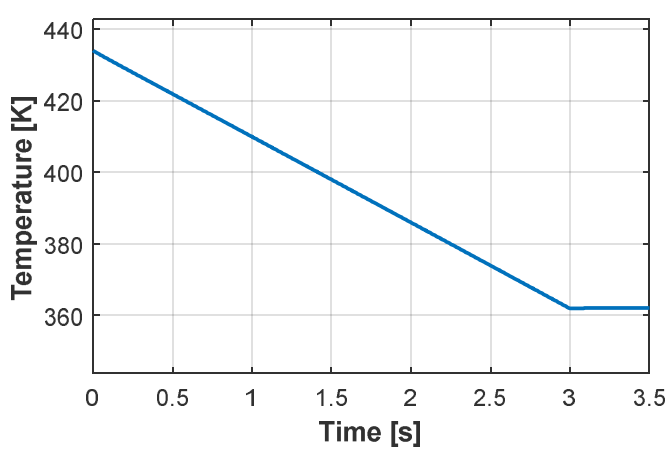

(b)

Figure 23: (a): Average static $P(b)$ : total T response in the pipe in FSP branch under scheduled conditions

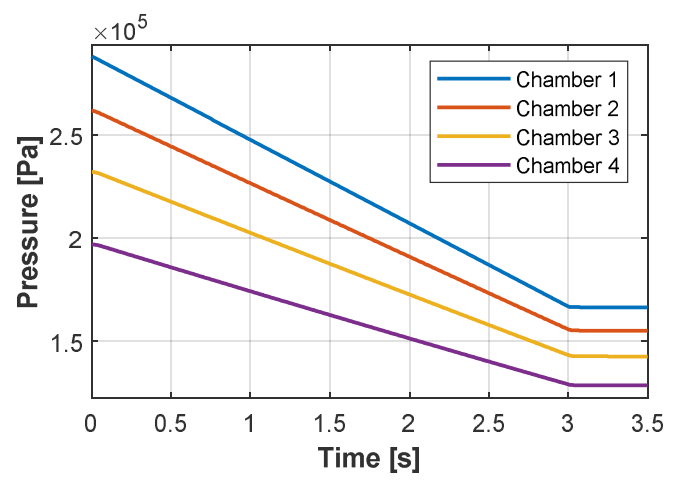

(a)

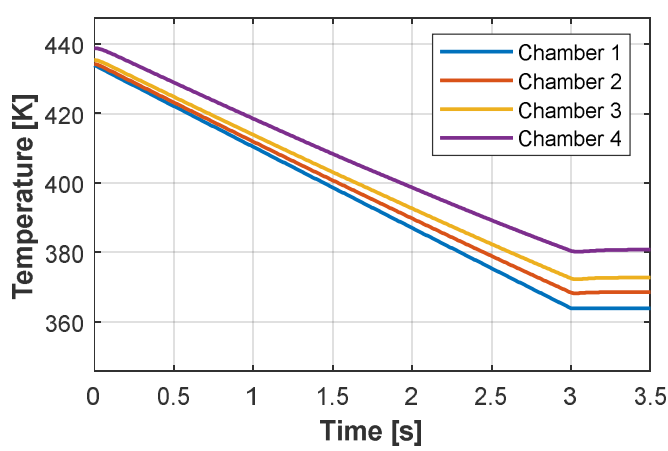

(b)

Figure 24: (a): Average static $\mathrm{P}$ (b): total $\mathrm{T}$ response in the ventilation branch chambers under scheduled conditions 


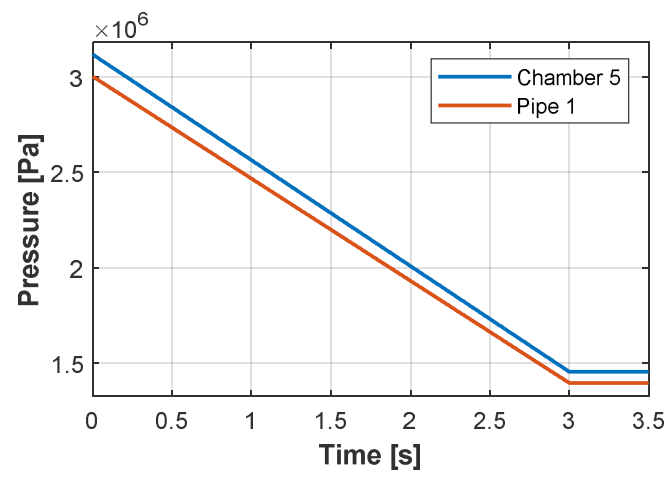

(a)

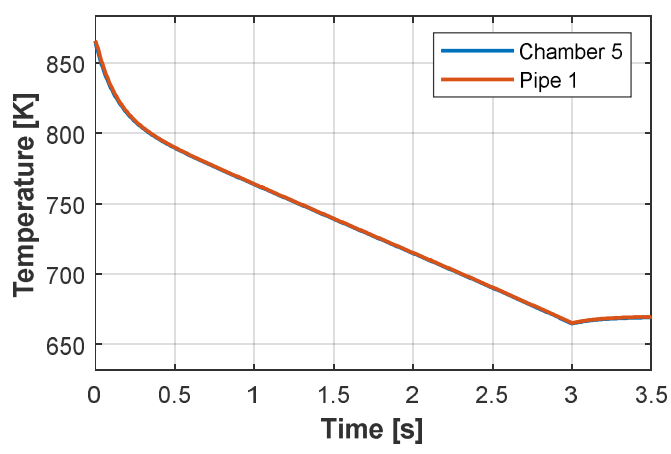

(b)

Figure 25: (a): Average static $\mathrm{P}(\mathrm{b})$ : total T response in the chambers in the pre-swirl nozzle and cooling passage of blade cooling under scheduled conditions

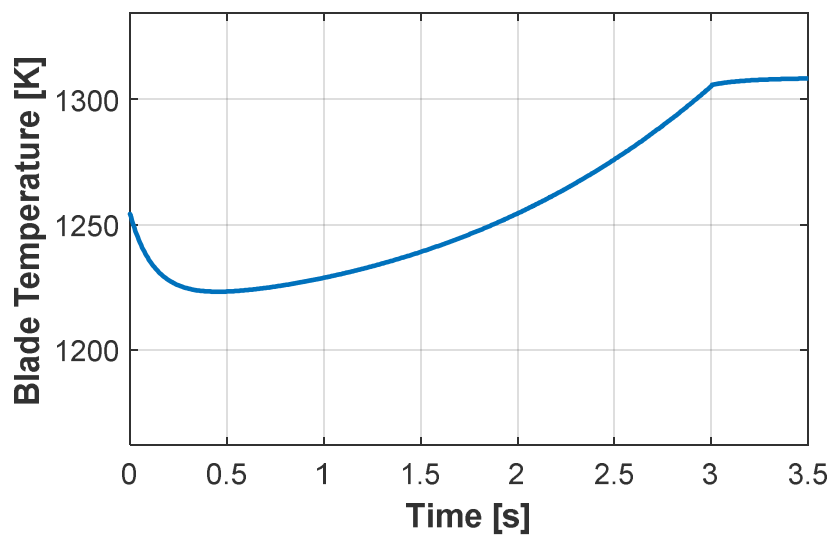

Figure 26 Blade temperature response in HPT branch under scheduled conditions

\subsection{Transient Vs steady-state flow characteristics}

Similar to the main gas path flow dimensionless analysis, the corrected mass flow and pressure ratio across the branches were chosen to represent the SAS flow behaviour. The flow transient characteristics (case II) were compared with the steady-state operational line (initial and final point - table 4) to indicate and how far the transient is from the steady state.

Both FSP and the ventilation branch show a smooth transient response almost parallel to the steady-state line (Figure 27). 


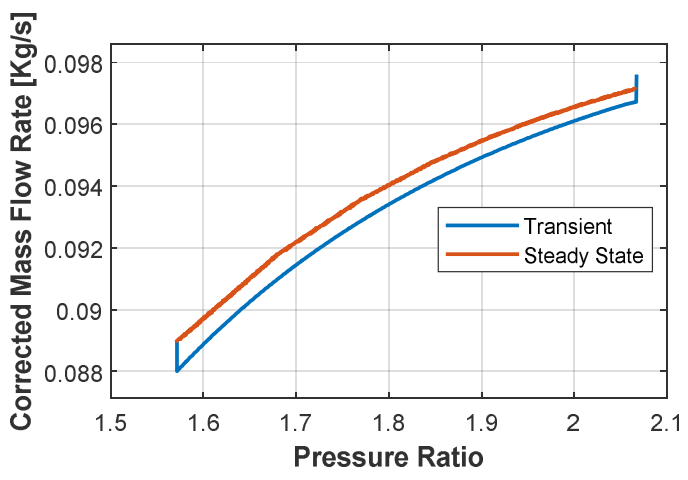

(a)

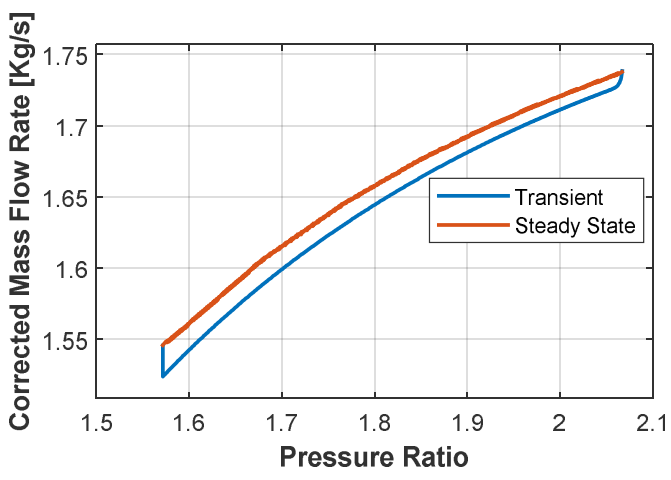

(b)

Figure 27: Transient performance of (a) FSP \& (b) ventilation branch

However, in the HPT rotor cooling branch, a rapid overshooting of mass flow at the starting point is observed (Figure 28). After the initial period, the transient operating point moves further away from the steady state operation line. When the boundary conditions are stabilized, the mass flow converges to the steady-state final value with a small overshooting at the end. This behaviour is explained by considering the high pressure and temperature at the boundaries (Figure 25), as well as the non-linear nature of this system.

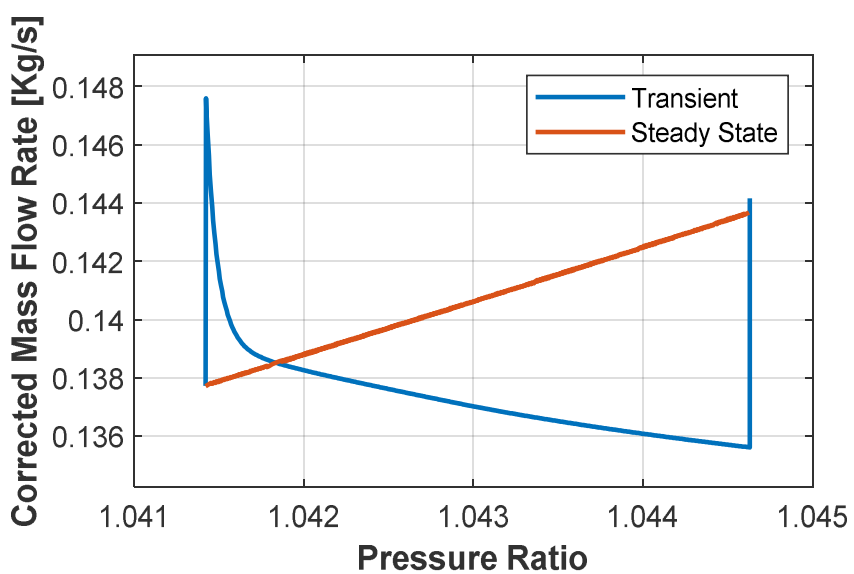

Figure 28: Transient performance of turbine blade cooling

\section{Conclusions}

An object-oriented SAS simulation platform has been developed by using a modular library for the different components of the SAS. A flow network simulation has been developed to replicate the transient behaviour of three main SAS branches in a 2-spool turbofan engine model under normal and extreme operating conditions. The performance of the critical element has been validated with public data and reasonable results have been produced using the platform developed in this paper. 
It was found that the developed framework is able to predict the behaviour of different components and branches in the SAS system at different transient operating conditions.

In case of any engine malfunction expressed by an abrupt change in the boundary operating conditions of the SAS, large flow reversal can take place in the different branches. Additionally, chocked flow can be observed at the rotor blade cooling branch.

The pressure response is much faster than the temperature. The settling time of pressure is in the order of 0.001 to $0.01 \mathrm{~s}$, and that of temperature is $0.1 \mathrm{~s}$ for the branches tested in this model. This can vary depending on the complexity of the SAS network (i.e. the number of branches and individual elements).

The flow performance under scheduled boundary variation (normal operating condition) transient test is much milder than the abrupt one. Consequently, in the normal operating condition, a steady-state simulation for the SAS is able to capture the system behaviour very well. However, the transient simulation would be very helpful in extreme operating conditions (i.e. shaft fracture, flameout, etc.). 


\section{Appendix}

1. Calculation of $M_{d}$ in equation (6) [14]:

The cumulative moment on the disc can be calculated as:

$$
M_{d}=\int_{r 1}^{r 2} \frac{d M_{d}}{d r} \mathrm{dr}
$$

The derivative of $M_{d}$ over radius $\mathrm{r}$ can be obtained as:

$$
\frac{d M_{d}}{d r}=\frac{5}{2} K C_{m, d i s k} \rho r^{4} \omega^{2} \operatorname{sgn}(\omega)
$$

where $C_{m, \text { disk }}$ is the free disc moment coefficient:

$$
C_{m, \text { disk }}=0.073 R e^{-0.2}
$$

and $\omega$ is the local relative angular velocity between the disc and the air. If the tangential velocity profile satisfies equation (1), $\omega$ can be calculated as:

$$
\omega(r)=\omega_{d}-K_{\varphi} r^{n-1}
$$

where $\omega_{d}$ is the disc angular velocity. $K_{\varphi}$ is a constant and $\mathrm{n}$ is the vortex index, indicating the vortex behaviour in the chamber [also used in equation (1)]. With this relative velocity being used, the model developed can be applied to either rotors or stators.

With equation (A-4), the cumulative moment can be obtained:

$$
M_{d}=\left\{\begin{array}{l}
\frac{1}{2} \mathrm{~K} C_{m, d i s k} \rho \omega^{2}\left(r_{2}^{5}-r_{1}^{5}\right) \quad \text { if } \mathrm{n}=1 \\
\frac{1}{2} \mathrm{KC}_{m}^{*} \rho \omega^{2}\left(r_{2}^{5}-r_{1}^{5}\right) \quad \text { if }-1 \leq \mathrm{n}<1
\end{array}\right.
$$

where $K$ is a constant adjusted to align with the physical system. $\mathrm{C}_{m}^{*}$ is determined as:

$$
\begin{aligned}
\mathrm{C}_{m}^{*}=C_{m, \text { disk }}(\mathrm{a} & +1)\left(\frac{\omega_{d}-\omega_{2}}{\omega_{2}}\right)^{2} \\
& \cdot\left(\frac{\operatorname{sgn}\left(\omega_{2}\right) F\left(\frac{\omega_{2}}{K_{\varphi}}, \frac{\omega_{d}}{K_{\varphi}}\right)-\operatorname{sgn}\left(\omega_{1}\right) F\left(\frac{\omega_{1}}{K_{\varphi}}, \frac{\omega_{d}}{K_{\varphi}}\right)}{\left(\frac{\omega_{d}-\omega_{2}}{K_{\varphi}}\right)^{a+3}}\right)
\end{aligned}
$$

where $\omega_{1}$ and $\omega_{2}$ are the angular velocity at the inner and outer cavity radius respectively. Function $F$ and constant $a$ are defined as:

$$
\begin{gathered}
F(x, y)=y^{2} \frac{\left((y-x)^{a+1}-y^{a+1}\right)}{a+1}-2 y \frac{\left((y-x)^{a+2}-y^{a+2}\right)}{a+2} \\
+\frac{\left((y-x)^{a+3}-y^{a+3}\right)}{a+3} \\
a=\frac{6-n}{n-1}
\end{gathered}
$$

2. Calculation of $M_{S}$ in equation (6) [14]: 


$$
\begin{gathered}
M_{S}=K_{S} C_{D} \frac{1}{2} \rho \omega|\omega|\left(2 \pi s_{\omega} r_{2}^{4}\right) \\
C_{D}=0.031(2 \pi \mathrm{Re})^{-1 / 7}
\end{gathered}
$$

where $K_{S}$ is constant adjusted to align with physical conditions. And $\mathrm{s}_{\omega}$ is the length of a portion of shroud which rotates in $\omega$.

3. Calculation of $Q_{\text {net }}$ in equation (5) [14]:

Heat input due to disc windage $Q_{n e t, \text { disc }}$ is modelled as:

$$
Q_{\text {net, disc }}=M_{d} \cdot \Omega_{d}
$$

$Q_{\text {net }}$ due to convection heat transfer is [4]:

$$
Q_{n e t, H T}=h_{a v} \cdot A_{s} \cdot\left(T_{s}-T_{r e f}\right)
$$

$A_{S}$ is the disc surface area. $T_{S}$ is the area weighted surface temperature and $T_{r e f}$ is the average temperature of the gas. $h_{a v}$ is the surface average heat transfer coefficient and its determination can be referenced to the appendix in reference [4].

Thus, the total $Q_{n e t}$ is a sum of the two part in equation (A-11) and (A-12):

$$
Q_{n e t}=Q_{n e t, \operatorname{disc}}+Q_{n e t, H T}
$$

4. Estimation of carry-over factor $K$ in equation (18) [18][19]:

$$
K_{c o}=\sqrt{\frac{1}{1-\frac{n_{t}-1}{n_{t}} \cdot \frac{c / p}{c / p+0.02}} \cdot \frac{n_{t}}{n_{t}-1}}
$$

where $n_{t}$ represents labyrinth teeth number, $\mathrm{c}$ represents teeth clearance and $\mathrm{p}$ represents teeth distance.

5. Calculation of discharge coefficient $C d$ in equation (15) [20]:

The determination of $C d$ is split in several steps. Firstly, a basic value is chosen based on engineering practice and then it is modified based on Reynolds number $(R e)$, corner radius $\left(r_{f}\right)$, length $(L)$ and relative tangential velocity $\left(U_{1}\right)$ effects. The basic value is chosen to be 0.5885 .

Firstly, the basic value is modified based on Reynolds number:

$$
C_{d: R e}=0.5885+\frac{372}{R e}
$$

Then, it is modified based on corner radius:

$$
C_{d: r}=1-\mathrm{f} \cdot\left(1-\mathrm{C}_{d: R e}\right)
$$


where $f$ is defined as:

$$
\mathrm{f}=0.008+0.993 \cdot \exp \left(-5.5 \cdot\left(r_{f} / \mathrm{D}\right)-3.5 \cdot\left(r_{f} / \mathrm{D}\right)^{2}\right)
$$

where $\mathrm{D}$ is the orifice diameter.

Then $C d$ is modified based on nozzle length $\mathrm{L}$ :

$$
C_{d: r, L}=1-\mathrm{g}\left(1-C_{d: r}\right)
$$

where function $\mathrm{g}$ is defined as:

$$
g=\left[1+1.3 \cdot \exp \left(-1.606 \cdot(\mathrm{L} / \mathrm{D})^{2}\right)\right] \cdot(0.435+0.021 \cdot \mathrm{L} / \mathrm{D})
$$

And finally, $C d$ is modified based on relative tangential velocity:

$$
C_{d: r, L, U}=C_{d: r, L} \cdot\left(C_{1}+C_{2} \cdot C_{3}\right)
$$

where constant $C_{1}, C_{2}$ and $C_{3}$ are defined as:

$$
\begin{gathered}
C_{1}=\exp \left(-R_{v}^{1.2}\right) \\
C_{2}=0.5 \cdot R_{v}^{0.6} \cdot\left(C_{d: r, L} / 0.6\right)^{-0.5} \\
C_{3}=\exp \left(-0.5 \cdot R v^{0.9}\right)
\end{gathered}
$$

where $R_{v}$ is defined as:

$$
R_{v}=\left(V_{\varphi} / V_{x}\right) \cdot\left(C_{d: r, L} / 0.6\right)^{-3}
$$

where $V_{\varphi}$ is the tangential velocity and $V_{x}$ is the axial velocity. 


\section{References}

[1] A. Moore, Gas Turbine Engine Internal Air Systems: A Review of the Requirements and the Problems, ASME 1975 Winter Annu. Meet. GT Pap. (1975) V001T01A001. doi:10.1115/75-WA/GT-1.

[2] Sultanian, B. (2018), Gas Turbines: Internal Flow Systems Modeling (Cambridge Aerospace Series), Cambridge: Cambridge University Press, doi:10.1017/9781316755686

[3] K.J. Kutz, T.M. Speer, Simulation of the Secondary Air System of Aero Engines, J. Turbomach. 116 (1994) 306. doi:10.1115/1.2928365.

[4] A. Alexiou, K. Mathioudakis, Secondary Air System Component Modeling for Engine Performance Simulations, J. Eng. Gas Turbines Power. 131 (2009) 031202. doi:10.1115/1.3030878.

[5] W. Che, S. Ding, C. Liu, A modeling method on aircraft engine based on the Component Method of secondary air system, Procedia Eng. 80 (2014) 258-271. doi:10.1016/j.proeng.2014.09.085.

[6] S. Brack, Y. Muller, Probabilistic Analysis of the Secondary Air System of a LowPressure Turbine, J. Eng. Gas Turbines Power. 137 (2014) 022602. doi:10.1115/1.4028372.

[7] Y. Muller, Secondary Air System Model for Integrated Thermomechanical Analysis of a Jet Engine, Vol. 4 Heat Transf. Parts A B. (2008) 1359-1374. doi:10.1115/GT200850078.

[8] S. Giuntini, A. Andreini, G. Cappuccini, B. Facchini, Finite element transient modelling for whole engine-secondary air system thermomechanical analysis, Energy Procedia. 126 (2017) 746-753. doi:10.1016/j.egypro.2017.08.231.

[9] H. Wu, P. Li, Y. Li, Simulation of unsteady state performance of a secondary air system by the 1D-3D-Structure coupled method, J. Therm. Sci. 25 (2016) 68-77. doi:10.1007/s11630-016-0835-1.

[10] L. Gallar, Gas Turbine Shaft Over-speed / Failure Performance Modelling, Ph.D thesis, Cranfield University, 2010. https://dspace.lib.cranfield.ac.uk/handle/1826/8353 .

[11] L. Gallar, C. Calcagni, V. Pachidis, P. Pilidis, Model - Part I: Tool Components Development and Validation, Asme Gt. (2009).

[12] C. Calcagni, L. Gallar, V. Pachidis, Development of a One-Dimensional Dynamic Gas Turbine Secondary Air System Model-Part II: Assembly and Validation of a Complete Network, in: 2010: pp. 435-443. doi:10.1115/GT2009-60051.

[13] D. May, J.W. Chew, Response of a Disk Cavity Flow to Gas Turbine Engine Transients, in: 2010: pp. 1113-1122. doi:10.1115/GT2010-22824.

[14] D. May, J.W. Chew, T.J. Scanlon, Prediction of De-Swirled Radial Inflow in Rotating Cavities With Hysteresis, in: 2013: pp. 2037-2046. doi:10.1115/GT2012-68647.

[15] M.J. Holland, T.F. Thake, Rotor blade cooling in high pressure turbines, J. Aircr. 17 (1980) 412-418. doi:10.2514/3.44668.

[16] M. Daly, B. Gunston, Jane's Aero Engines, 2nd ed., IHS Jane's, 2010. 
[17] Rolls-Royce, The Jet Engine, 5th ed., Rolls Royce plc, 1996.

[18] B. Hodkinson, Estimation of the Leakage through a Labyrinth Gland, Proc. Inst. Mech. Eng. 141 (1939) 283-288. doi:10.1243/PIME_PROC_1939_141_037_02.

[19] H. Zimmermann, K.H. Wolff, Air System Correlations: Part 1 - Labyrinth Seals, (1998) V004T09A048. http://dx.doi.org/10.1115/98-GT-206.

[20] W.F. McGreehan, M.J. Schotsch, Flow Characteristics of Long Orifices With Rotation and Corner Radiusing, J. Turbomach. 110 (1988) 213. doi:10.1115/1.3262183.

[21] P.R. Farthing, J.W. Chew, J.M. Owen, The Use of Deswirl Nozzles to Reduce the Pressure Drop in a Rotating Cavity With a Radial Inflow, J. Turbomach. 113 (1991) 106-114. http://dx.doi.org/10.1115/1.2927727.

[22] W. L. Macmillan, Development of a Modular Type Computer Program for the Calculation of Gas Turbine Off-Design Performance, PhD Thesis, Cranfield Inst. of Technology, UK, 1974. https://dspace.lib.cranfield.ac.uk/handle/1826/7401.

[23] Th. Nikolaidis, "The TURBOMATCH scheme for Gas Turbine Performance Calculations," unpublished software manual, 2017, Cranfield University, UK.

[24] P.R.N. Childs, Mechanical Design, 2nd ed., Elsevier Ltd., 2004.

[25] C. Soria, Gas Turbine Shaft Over-speed / Failure Performance Modelling, Ph.D thesis, Cranfield University, 2014. http://dspace.lib.cranfield.ac.uk/handle/1826/12131. 


\section{Transient modelling and simulation of gas turbine secondary air system}

Nikolaidis, Theoklis

Elsevier

Nikolaidis T, Wang H, Laskaridis P. (2020) Transient modelling and simulation of gas turbine secondary air system. Applied Thermal Engineering, Volume 170, April 2020, Article number 115038 https://doi.org/10.1016/j.applthermaleng.2020.115038

Downloaded from Cranfield Library Services E-Repository 